\title{
Michael DONDERER
}

\section{ANTIKE BILDHAUERSIGNATUREN - WO MAN SIE NICHT ERWARTEN WÜRDE}

»Nachdem er (Sostratos) das Werk errichtet hatte, ließ er seinen Namen innen auf das Mauerwerk schreiben. Er ließ ihn mit Mörtel bestreichen und, nachdem er ihn so hatte verbergen lassen, ließ er den Namen des damals regierenden Königs daraufschreiben; denn er wußte wohl - was dann auch eintrat -, daß nach ganz kurzer Zeit die Buchstaben zusammen mit dem Mörtel herabfallen würden und (folgende Inschrift) zum Vorschein kommen werde: Sostratos, der Sohn des Dexiphanes, aus Knidos stammend, (widmet es) den göttlichen Rettern zum Wohle der Seefahrer. $\aleph^{1}$ So Lukian (Hist. conscr. 62) zur Inschrift des Sostratos ${ }^{2}$ am Pharos von Alexandria. Zwar tendiert die neuere Forschung dahin, die überlieferte Anekdote für unglaubwürdig zu halten ${ }^{3}$, doch zeigt die Erzählung immerhin, daß eine versteckt angebrachte Signatur oder Stifterinschrift ${ }^{4}$ damals vorstellbar war. Bemerkenswert zahlreich sind auf Architekturgliedern Steinmetzinschriften, die vor dem Versetzen entweder noch hätten abgearbeitet werden müssen oder die nach dem Versetzen nicht mehr lesbar waren ${ }^{5}$. Genannt sei hier nur der spätantike Text auf einer Archivoltenrückseite aus Libyen ${ }^{6}$. Doch gibt es das bisher nicht beachtete Phänomen der abseitig oder versteckt angebrachten Künstlersignaturen auch auf Skulpturen?.

Bekanntlich befindet sich die Namensnennung griechischer Bildhauer und Bronzegießer für gewöhnlich auf den Basen, meist unterhalb der Votiv- oder Ehreninschrift ${ }^{8}$. Nur ausnahmsweise brachten archaische Künstler ihre Signatur am Werk selbst an ${ }^{9}$, häufiger wurde dieser Usus im

Für Hinweise und die Überlassung von Photos sei an dieser Stelle Chr. Börker (Erlangen/Berlin), G. Koch (Marburg), M. Prange (Hamburg) und J. Raeder (Kiel) vielmals gedankt. Zitiert wird nach den Richtlinien der ÖJh 69, 2000, 357 ff. (htt://www.oeai.at/publik/autoren.html); zusätzlich verwendet werden folgende Abkürzungen:

Loewy E. Loewy, Inschriften griechischer Bildhauer (1885).

KLA Künstlerlexikon der Antike I (2001).

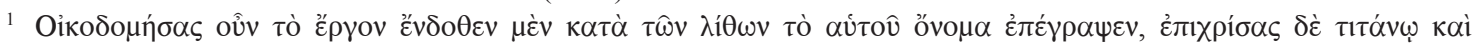

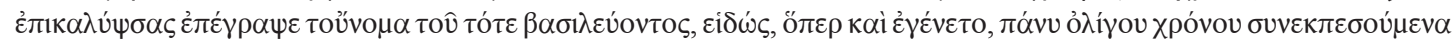

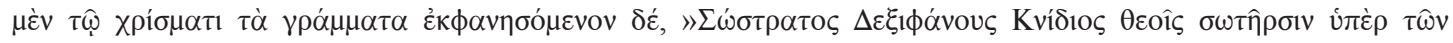
$\pi \lambda \omega i \zeta \zeta o \mu \varepsilon ́ v \omega v . \ll$

2 Zu diesem allgemein P. Perdrizet, Sostrate de Cnide, architecte du Phare, REA 1, 1898, 261 ff.; F. Heichelheim in: RE Suppl. VII (1940) 1221 f. s. v. Sostratos 11a; A. Adriani, Repertorio d'arte dell'Egitto greco-romano, Serie C I (1966) 103 ff.; EAA VII (1966) 416 s. v. Sostratos 4; W. Müller, Architekten in der Welt der Antike (1989) 96. 204 f.; M. Donderer, Die Architekten der späten römischen Republik und der Kaiserzeit. Epigraphische Zeugnisse (1996) 35. 65 Anm. 229; 79 f.; M.-Chr. Hellmann, L'architecture grecque I (2002) $52 \mathrm{f}$.

3 P. M. Fraser, Ptolemaic Alexandria I (1972) 19; F. Chamoux in: Le monde grec. Hommages à Claire Préaux (1978) 220 f.; T. L. Shear, Jr., Kallias of Sphettos and the Revolt of Athens in 286 B.C. (1978) 23; A. Bernand, ZPE 113, 1996, 86 f.; W. Ekschmitt, Die sieben Weltwunder ${ }^{10}$ (1996) 189 f.; G. Grimm, Alexandria. Die erste Königsstadt der hellenistischen Welt (1998) 45; W. Huß, Ägypten in hellenistischer Zeit (2001) 219.

4 Umstritten bleibt nämlich bis heute, zumal die Schriftquellen diesbezüglich nicht eindeutig sind, ob Sostratos Architekt und Stifter zugleich oder nur der Stifter des Pharos war.

5 M. Donderer, Gymnasium 102, 1995, 110 (Lit.).

${ }^{6}$ [Te]nteithanus (s)cyrbsit (sic) / bibas ma(gi)ster Kartitis (Tripolis, Archäolog. Museum): M. Guarducci, QuadALibya 7, 1975, 104 f. 129 f. (Lit.) Abb. 35. 75. 115. 116.

7 Nur kurz erwähnt von I. Calabi Limentani, Studi sulla società romana. Il lavoro artistico (1958) 87.

8 Loewy; J. Marcadé, Recueil des signatures des sculpteurs grecs I (1953); II (1957).

9 M. Donderer, Bildhauersignaturen auf griechischer Rundplastik, ÖJh 65, 1996, 87 ff. 
Hellenismus ${ }^{10}$, während es in der Kaiserzeit die Regel war ${ }^{11}$. Die Art der Aufstellung - ohne Basis bzw. in privatem Ambiente ohne weitere Inschrift - sowie weite Transportwege mögen die Ursache für diesen Wechsel gewesen $\operatorname{sein}^{12}$. Entscheidend aber ist: In den allermeisten Fällen erschien der Künstlername auf der Ansichtsseite und konnte somit gar nicht übersehen werden, zumal wenn er noch farblich hervorgehoben war. Überraschend ist jedoch, daß gelegentlich auch unvermutete Anbringungsorte zu belegen sind.

Nahezu ausnahmslos besitzen nackte oder nur leicht bekleidete Statuen aus Marmor eine Stütze - meist in Form eines Baumstrunkes -, die seit dem Hellenismus der bevorzugte Anbringungsort für Signaturen $w^{13}{ }^{13}$. Doch bemerkenswerterweise diente dazu nicht nur die Hauptansichtsseite, sondern gelegentlich auch die Nebenseite, so daß sich der Text nur beim Umschreiten des Werkes erschloß.

In der Formulierung eindeutig sind die Signaturen zu Seiten der Baumstütze einer Heraklesstatue aus Ptolemais ${ }^{14}$ sowie einer wohl männlichen Statue aus Verona ${ }^{15}$. Anzuschließen ist eine Panstatue aus Lanuvium ${ }^{16}$, deren stilistisch abweichendes Pendant eine leicht erweiterte unbeschädigte Signatur nach dem Vorbild der motivgleichen Statue aufweist $t^{17}$. Allerdings ist nicht sicher, ob die Inschrift wirklich antik bzw. zugehörig ist ${ }^{18}$. Die ausdrückliche Angabe des Freigelassenenstatus im griechischen Kontext ${ }^{19}$ ist nämlich bis heute singulär; außerdem gibt es keinen Beleg dafür, daß auf einer Statuenkopie die ursprüngliche Signatur ebenfalls übernommen worden wäre ${ }^{20}$.

Ebenfalls in den hier behandelten Zusammenhang gehört die bei Frascati gefundene Ama$z^{2} e^{21}$ (Abb. 1), die lediglich einen Namen im Nominativ auf der Nebenseite des Baumstumpfes trägt $^{22}$, erweitert durch den Buchstaben $\mathrm{N}$ innerhalb eines Kreises ${ }^{23}$ (Abb. 2). Trotz Fehlens des

10 Donderer (Anm. 9) 94 ff.

11 G. M. A. Richter, Three Critical Periods in Greek Sculpture (1951) 45 ff.

12 M. Donderer, ZPE 73, 1988, 66.

13 Loewy VII.

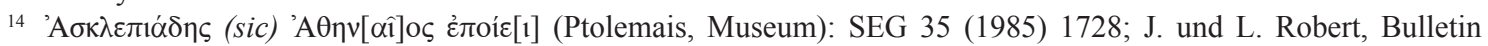
Epigraphique 1964, 589; E. Fabbricotti in: Cyrenaica in Antiquity, Colloquium Cambridge 1983 (1985) $219 \mathrm{ff}$. Taf. 18, 1; G. Bröker in: KLA 99 s. v. Asklepiades V (Lit.).

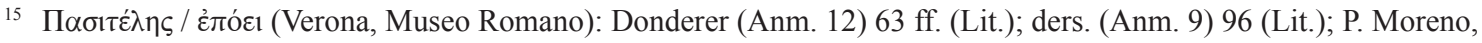
Scultura ellenistica II (1994) 740 Abb. 909; ders. in: EAA 2. Suppl. IV (1996) 268 f. s. v. Pasiteles Abb. 268; M. Sève, Bulletin Epigraphique 1997, 80; P. Moreno, Archeo 18 Nr. 207, 2002, 98 Abb. - Weiterhin fälschlich auf Praxiteles bezogen: A. Corso, NumAntCl 25, 1996, 144 f. Abb. 20. 21; ders., RdA 22, 1998, 36; F. Rebecchi in:

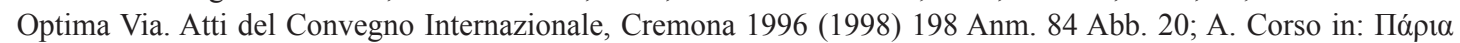
$\lambda i \theta$ os (2000) 234 Abb. 18. 19.

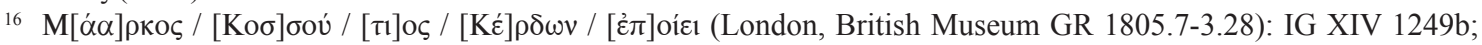
Loewy 263 f. Nr. 376b; Richter (Anm. 11) 46 Abb. 85; M. Torelli, MemAmAc 36, 1980, 315; A. Dostert in: Standorte. Kontext und Funktion antiker Skulptur (Ausstellungskat. Berlin 1994/95 [1995]) 424; M. Fuchs, In hoc etiam genere Graeciae nihil cedamus (1999) 64 ff. Abb. 2 (Lit.) Taf. 55. 57. 59; S. Ingegneri, Daidalos 3, 2001, 268 Taf. 44; R. Vollkommer in: KLA 147 f. s. v. Cossutius Cerdo (Lit.).

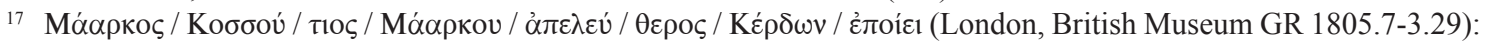
IG XIV 1249a; Loewy 263 f. Nr. 376a; Richter (Anm. 11); Torelli (Anm. 16); Dostert (Anm. 16) 423 ff. Nr. D 20 (Lit.) Abb.; Fuchs (Anm. 16) Taf. 54. 56. 58; Ingegneri (Anm. 16) 268 Taf. 45; Vollkommer (Anm. 16).

18 Belege bei Loewy 264.

19 Vgl. allerdings einen lateinischen Beleg aus der Umgebung Roms: s. u. Anm. 64.

20 D. Arnold, Die Polykletnachfolge (1969) 248; Chr. Vorster in: H. Beck - P. C. Bol (Hrsg.), Polykletforschungen (1993) 202; D. Kreikenbom in: Hellenistische Gruppen. Gedenkschrift A. Linfert (1999) 344 mit Anm. 21. Dagegen zu Recht Fuchs (Anm. 16) 66.

${ }_{21}$ R. Bol, Amazones Volneratae (1998) 188 Nr. II (Lit.) Taf. 52. 53. 80. $81 \mathrm{a} ; 135 \mathrm{f}$.

$22 \Sigma \omega \sigma \mid \kappa \lambda \hat{\eta}(\varsigma) / N$ (Rom, Kapitolin. Museen): IG XIV 148*; Loewy 290 f. Nr. 434; H. von Steuben in: Griechische Klassik, Vorträge Blaubeuren 1991 (1994) 276 ff. Taf. 20; P. Liverani, BMusRom N. S. 10, 1996, 98 ff. Abb. 1; F. P. Arata, BullCom 99, 1998, 176 ff. Nr. 18 Abb. 23. 24.

23 Dabei dürfte es sich kaum um eine Zahlengabe (so jedoch L. Moretti, Inscriptiones Graecae Urbis Romae IV [1990]

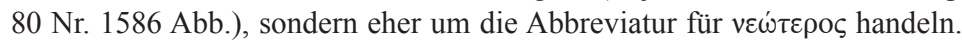




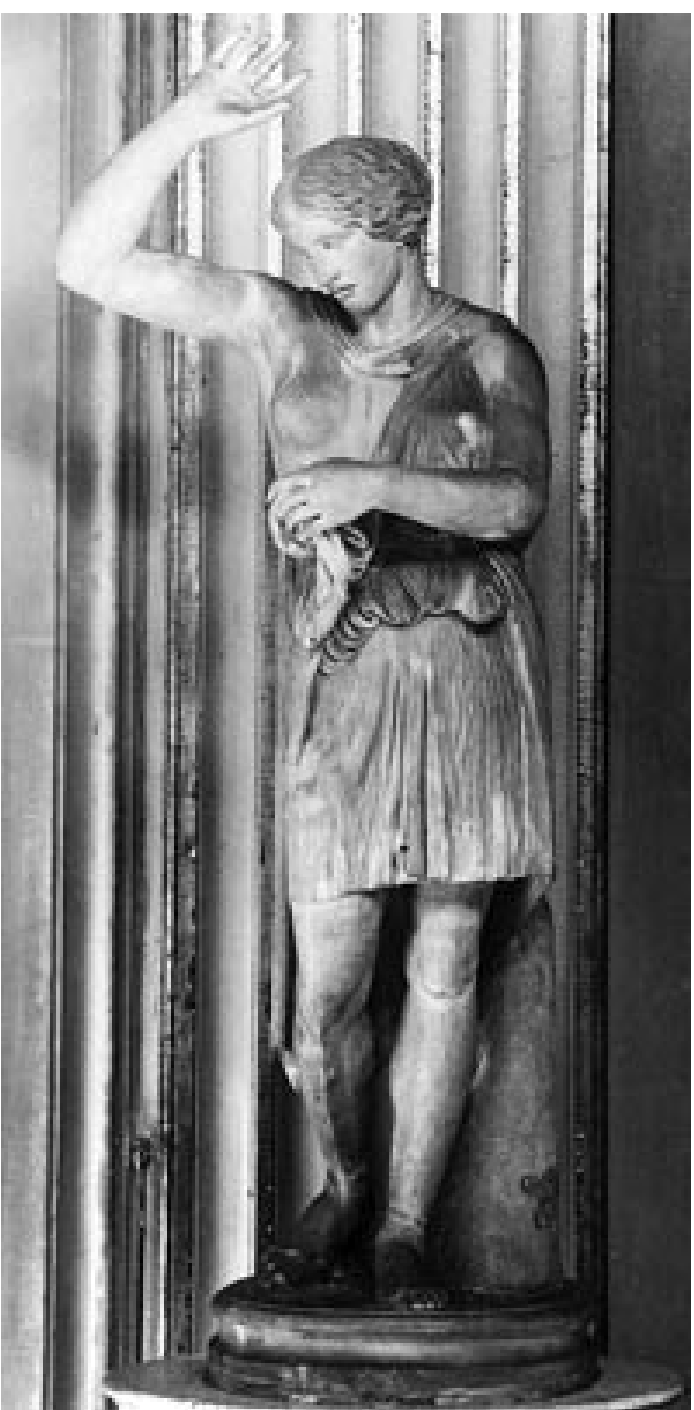

1 Amazone aus der Umgebung von Frascati

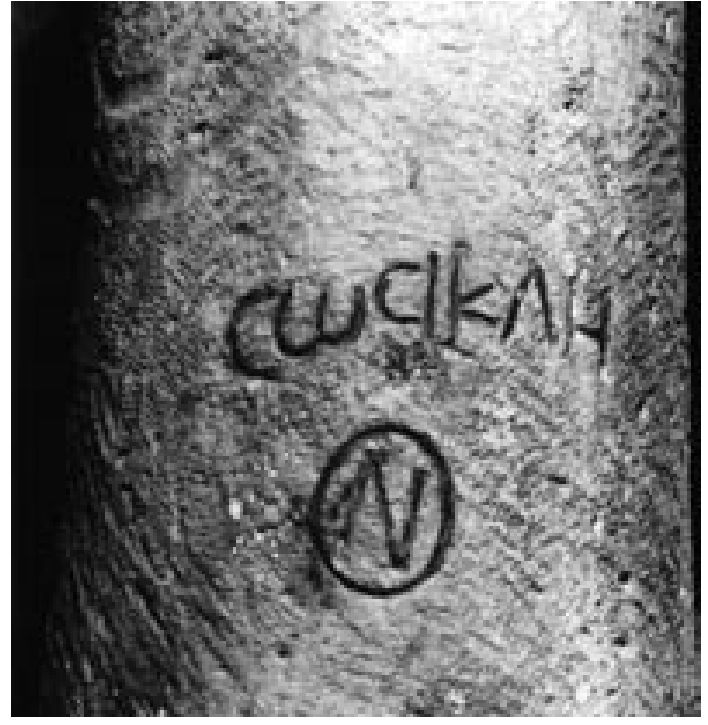

2 Signatur des Sosikles

Verbums als Determinativ muß eine Signatur vorliegen, da eine Besitzer- oder Stifterangabe an dieser Stelle und in diesem Zusammenhang keinen Sinn ergibt. Als Parallele sei auf die beiden Kentauren aus der Villa Hadriana bei Tivoli verwiesen, auf deren profilierten Plinthen jeweils die Doppelsignatur der Namen im Nominativ, erweitert um das für die Deutung wichtige Ethnikon, erscheint ${ }^{24}$.

Auf der Seitenwandung des Kalathos einer Karyatide, gefunden bei Rom, ist die Signatur zweier Bildhauer zu lesen ${ }^{25}$. In diesem Fall ist davon auszugehen, daß die Buchstaben nicht nur wegen der Höhe ihrer Anbringung, sondern auch aufgrund der Tatsache kaum wahrgenommen werden konnten, daß sie meist infolge des aufliegenden Gebälks im Schatten lagen.

Vom Celio in Rom stammt eine nackte Aphroditestatue, Typus Kapitol26 (Abb. 3). An der nach außen gekehrten Seitenfläche des Schmuckkästchens unterhalb des Gewandes zur Linken

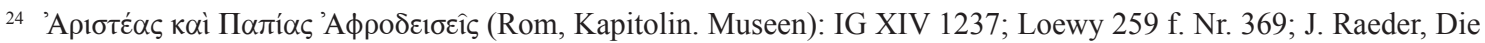
statuarische Ausstattung der Villa Hadriana bei Tivoli (1983) 63 ff. Nr. I 46.47 (Lit.); V. Saladino in: In Memoria di Enrico Paribeni II (1998) 384 ff. Taf. 110, 2; 112, 1; 113, 1; M. Bergmann, Chiragan, Aphrodisias, Konstantinopel (1999) 37. 55. 62 Taf. 73. 74, 2. 4; 75, 1. 2; H. Gregarek, KölnJb 32, 1999, 113. 237 f. Nr. D 166. 169 Abb. 82. 83; Arata (Anm. 22) 206 ff. Nr. 78. 79 Abb. 66 ff.; G. Morawietz, Der gezähmte Kentaur (2000) 50 f. 67.89 ff. 94 ff. 111 ff. 126. 129 Abb. 31 ff.; G. Bröker in: KLA 81 s. v. Aristeas I (Lit.).

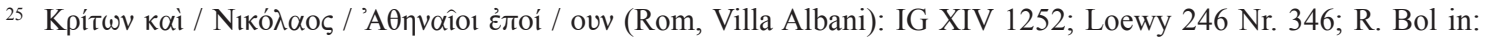
Forschungen zur Villa Albani, Katalog der antiken Bildwerke II (1990) 90 ff. Nr. 178 (Lit.) Taf. 46 ff.; Moretti (Anm. 23) 69 Nr. 1570; R. Vollkommer in: KLA 432 s. v. Kriton IV.

26 O. Vasori in: Museo Nazionale Romano. Le sculture I 1 (1979) 109 ff. Nr. 81 (Lit.); A. Delivorrias in: LIMC II (1984) 54 s. v. Aphrodite 422 Taf. 40; R. R. R. Smith, Hellenistic Sculpture (1991) 80 Abb. 101; A. Corso, NumAntCl 21, 1992, 142 ff. Abb. 8; P. Moreno, Scultura ellenistica (1994) I 109 f. 498; II 657 f. 668.716 Abb. 797; Chr. M. Havelock, The Aphrodite of Knidos and Her Successors (1995) 80 Abb. 22; A. Stewart, Art, Desire and the Body in Ancient Greece (1997) 106 Abb. 64. 


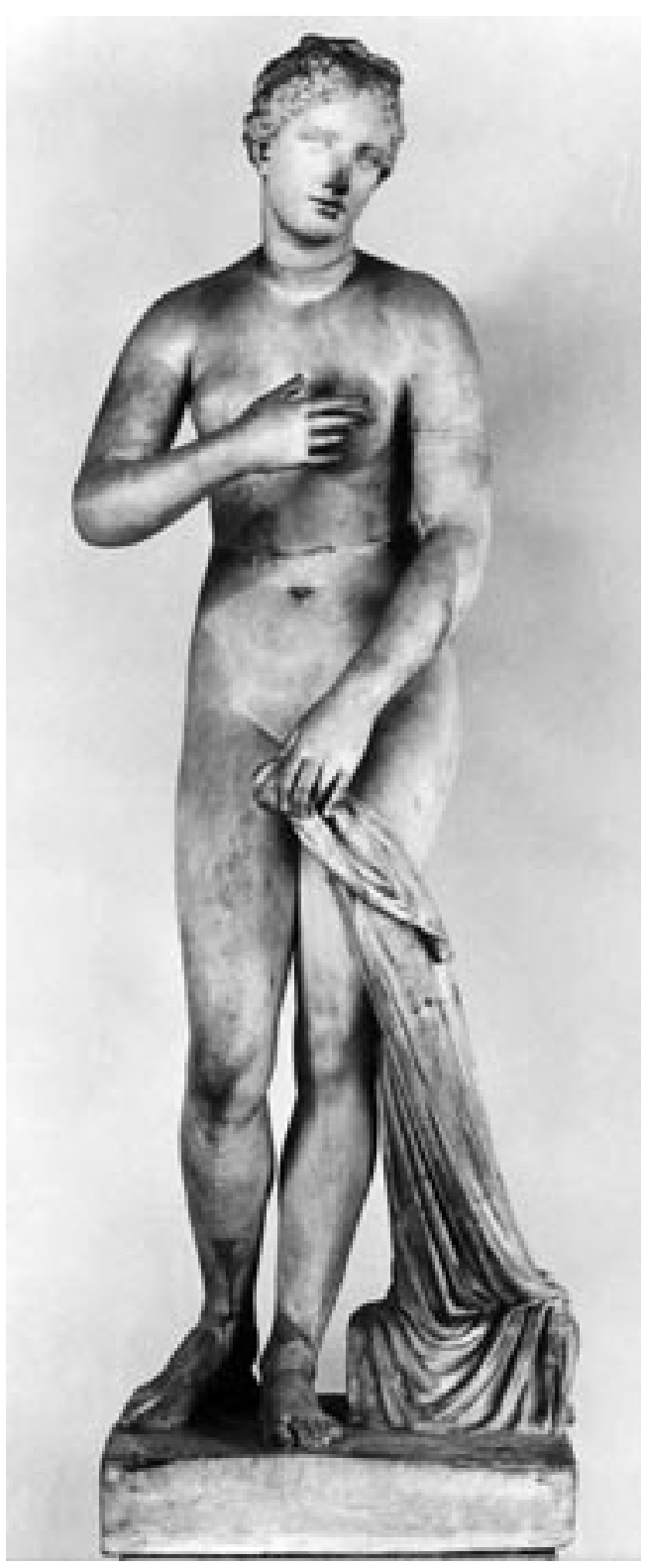

3 Aphrodite aus Rom

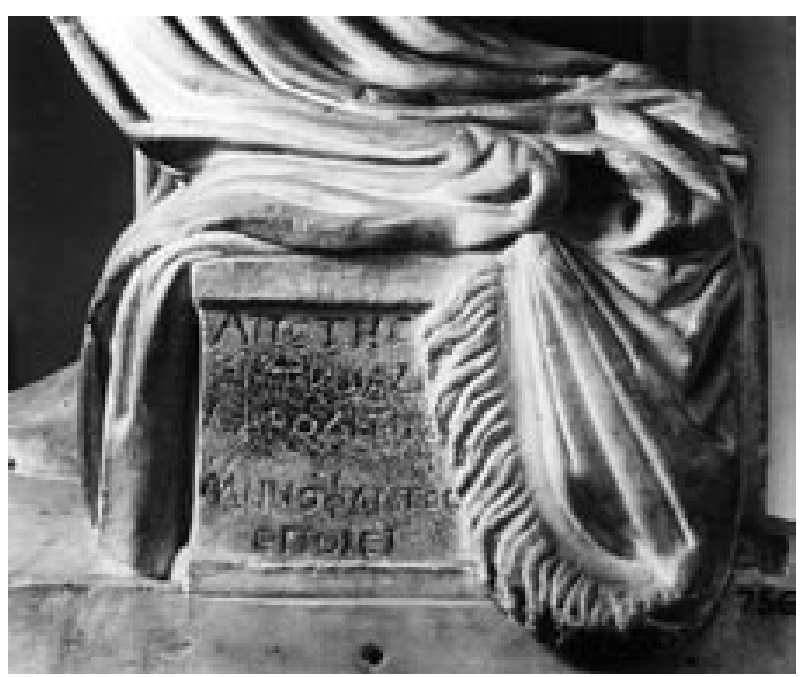

4 Signatur des Menophantos

der Göttin prangt nicht nur der Bildhauername, sondern auch die bis heute singuläre zusätzliche Aussage über das dieser Kopie zugrundeliegende Vorbild $^{27}$ (Abb. 4). Sowohl die Existenz der Schatulle, die in der Frontalansicht unter der als Stütze dienenden Stoffülle nur zu erahnen war, wie auch die textliche Information erschließen sich für den Betrachter jedoch erst beim Umschreiten der Plastik - aber gerade dazu lud das Thema ja bekanntermaßen ein: die knidische Aphrodite des Praxiteles war für ihre Rückansicht berühmt ${ }^{28}$. Außerdem lassen sich offene Rundbauten nachweisen, in denen Aphroditestatuen von allen Seiten begutachtet werden konnten ${ }^{29}$.

Allerdings ließen sich Freiplastiken nicht nur an den Nebenseiten der Stützen signieren, sondern erstaunlicherweise auch auf deren Rückseite. Als Belege können der Bronzetorso eines unbekleideten

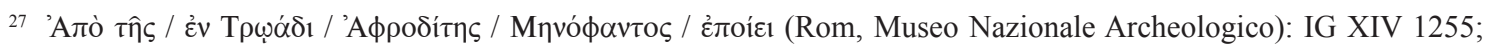
Loewy 264 Nr. 377; Moretti (Anm. 23) 75 Nr. 1577 Abb.

28 Plin. nat. 36, 21 = Overbeck, Schriftquellen Nr. 1227; A. Corso, Prassitele, Fonti epigrafiche e letterarie. Vita e opere I (1988) 76 ff. - Lukian. am. 13 f. = Overbeck, Schriftquellen Nr. 1234. - Delivorrias (Anm. 26) 49 f.; Corso a. O. $127 \mathrm{ff}$.

29 Rundtempel auf der Thalamegos des Ptolemaios IV.: M. Pfrommer in: Basileia. Die Paläste der hellenistischen Könige (1996) 97 ff. Abb. 2; ders., Nürnberger Blätter zur Archäologie 13, 1996/97, 82 Abb. 14; ders., Alexandria. Im Schatten der Pyramiden (1999) 108 ff. Abb. 148. Frontispiz. - Rundtempel in der Villa Hadriana bei Tivoli: Raeder (Anm. 24) 95 Nr. I 97 Taf. 17; H. Mielsch, Die römische Villa (1987) 112 f. Abb. 78; M. Weber, Baldachine und Statuenschreine (1990) 108. - Wohl nicht der Aphrodite zuzuschreiben ist ein hellenistischer Rundtempel in Knidos: B. Vierneisel-Schlörb, Klassische Skulpturen des 5. und 4. Jahrhunderts v.Chr., Glyptothek München, Katalog der Skulpturen II (1979) 326 f.; Delivorrias (Anm. 26) 49 f.; H. von Steuben, IstMitt 39, 1989, 541 f.; Weber a. O. 106 ff.; Havelock (Anm. 26) 60 f. Abb. 15; H. Bankel, AA 1997, 51 ff.; Chr. Bruns-Özgan, Knidos. Ein Führer durch die Ruinen (2002) 73 ff. 


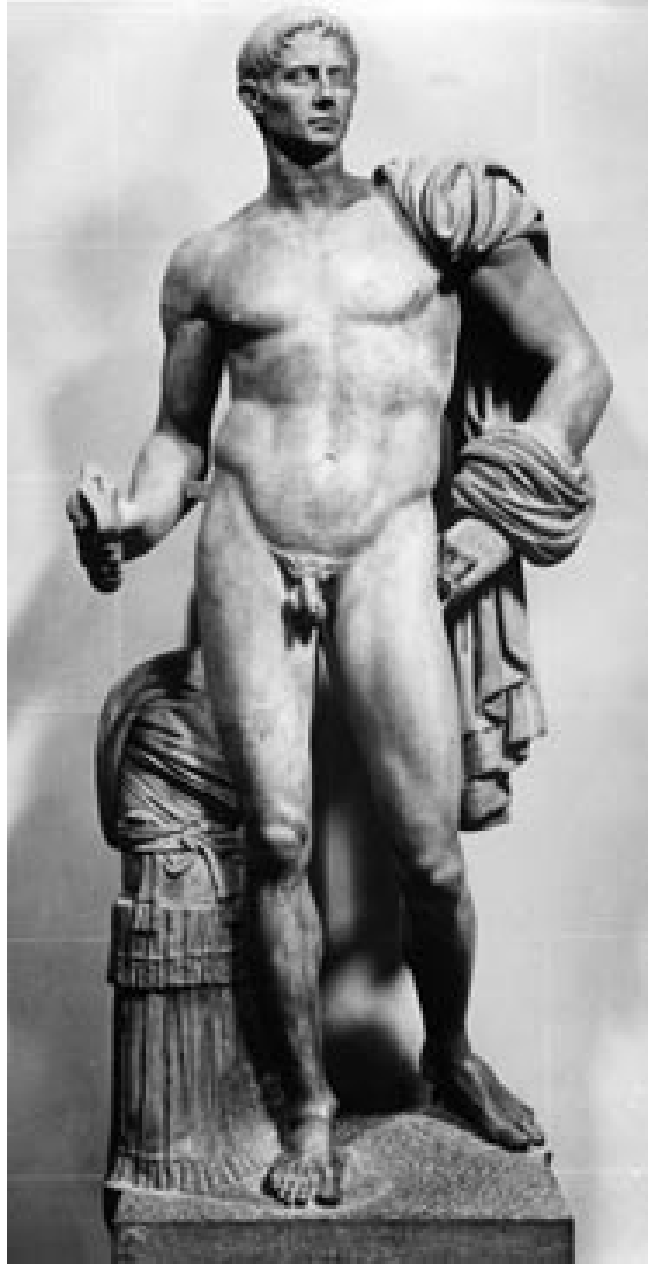

5 Porträtstatue aus der Umgebung von Tusculum

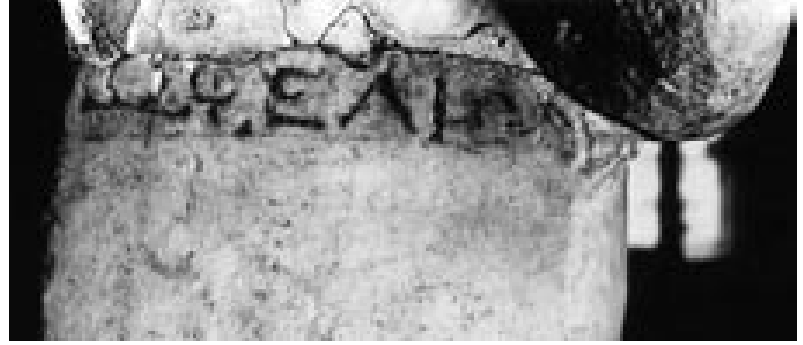

6 Signatur des Ophelion

Mannes unbekannter Provenienz $z^{30}$ sowie die männliche Idealstatue mit Porträtkopf dienen, die bei Tusculum zutage gekommen ist ${ }^{31}$ (Abb. 5. 6).

Einen Sonderfall stellt die nur in ihrem unteren Teil erhaltene männliche Mantelstatue des ausgehenden 2. Jahrhunderts v. Chr. aus Samos dar. An sich hätte sie keiner Stütze bedurft, doch entschied sich der Bildhauer offensichtlich für sie, da er auf ihr seinen Namen einmeißeln konnte ${ }^{32}$. Möglicherweise war das Werk selbst in dieser Hinsicht tabu, gehört die Plastik doch zu den ältesten Opera nacharchaischer Zeit, die selbst eine Signatur tragen ${ }^{33}$. In Kauf genommen wurde dabei, daß die Stütze mit der Inschrift an der rechten Nebenseite plaziert wurde, so daß die Signatur nur lesbar war, wenn der Betrachter auch die rechte Seite der Plastik in Augenschein nahm.

Offenbar erst im Laufe des 1. Jahrhunderts v. Chr. konnten Bildhauersignaturen Gewandpartien bekleideter Statuen eingeschrieben werden. Meist erscheinen sie in Kniehöhe, so daß sie zu erkennen

waren, wenn die Skulptur jeweils auf einem ca. $1 \mathrm{~m}$ hohen Sockel stand. Allerdings ist fraglich, ob sie deutlich zu lesen waren, wenn die Gewandstatuen, wie üblich, bemalt waren ${ }^{34}$, denn es läßt sich nicht mehr klären, ob die Inschriften ehedem farblich abgesetzt waren.

Die bisher älteste Signatur (noch erste Hälfte des 1. Jahrhunderts v. Chr.?) auf einer Gewandpartie gehört zu einer in mindestens zwei Teilen gearbeiteten Statue aus Piacenza, von der

30 "A $\tau \tau \alpha$ (amerikanische Privatsammlung): C. C. Mattusch, The Fire of Hephaistos (Ausstellungskat. Cambridge, Mass. 1996 [1996]) 349 f. Nr. 55 Abb.; M. Sève, Bulletin Epigraphique 1997, 85. - Zum Namen vgl. L. Barkóczi - A. Móczy, Die römischen Inschriften Ungarns I (1972) 94 Nr. 103.

31 ' $\Omega \phi \varepsilon \lambda i \omega_{v} /$ ['A] $\rho 1 \sigma \sigma \tau o v i ́ \delta \alpha$ (Paris, Musee du Louvre): IG XIV 1277; Loewy 289 f. Nr. 432; Richter (Anm. 11) 54 Abb. 112; D. E. E. Kleiner - F. S. Kleiner, AA 1975, 253 f. 262 Nr. 21; 257 Abb. 3; K. de Kersauson, Catalogue des portraits romains de Musée du Louvre I (1986) 78 Nr. 33 (Lit.) Abb.

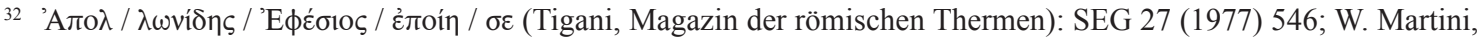
AA 1972, 292 ff. Abb. 11 ff.; Donderer (Anm. 9) 100 (Lit.) Abb. 11. 12; R. Vollkommer in: KLA 67 f. s. v. Apollonides III.

33 Donderer (Anm. 9) 100 ff.

34 Vgl. allgemein P. Reuterswärd, Studien zur Polychromie der Plastik. Griechenland und Rom (1960); V. Brinkmann - U. Koch-Brinkmann, Der prächtige Prinz (2002); B. Freyer-Schauenburg, AM 117, 2002, 289 ff.; V. Brinkmann, Die Polychromie der archaischen und frühklassischen Skulptur (2003); Bunte Götter (Ausstellungskat. München 2003/2004 [2003]); ClassiColor. Farven i antik skulptur (2004). 


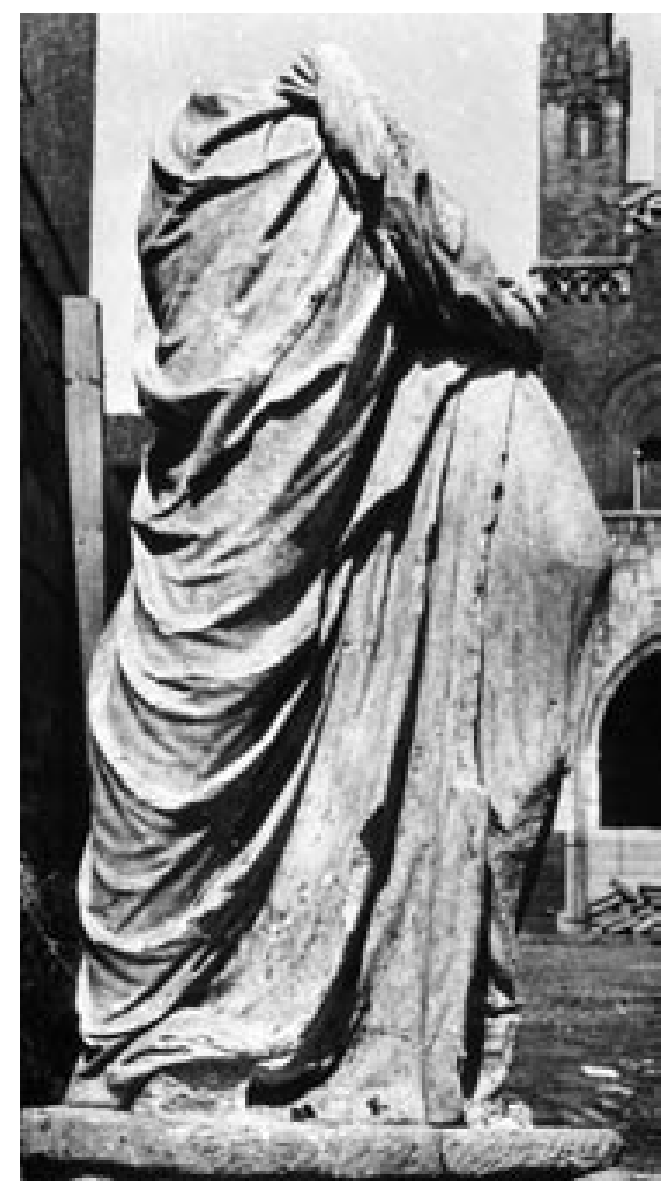

7 Statue aus Piacenza

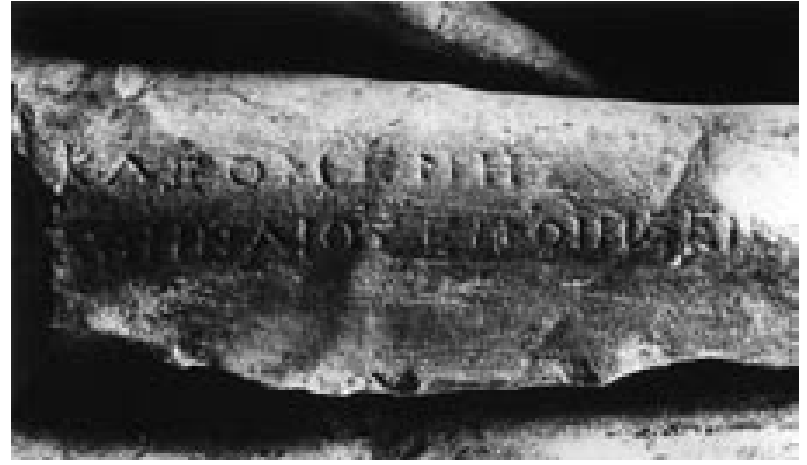

8 Signatur des Kleomenes

sich nur die untere Partie erhalten hat ${ }^{35}$ (Abb. 7). Umstritten ist, ob es sich um eine Darstellung Apolls oder um eine Frauenstatue handelt ${ }^{36}$. Die Künstlerinschrift befindet sich wiederum auf der rechten Nebenseite ${ }^{37}$ (Abb. 8).

Bei zwei Sitzstatuen erscheint der Künstlername bereits in der Archaik auf der vom Betrachter aus rechten Seitenfläche des Sitzmöbels ${ }^{38}$. Einen weiteren Beleg, allerdings erst aus der frühen Kaiserzeit, stellt eine stark beschädigte Sitzstatue aus dem Fayum dar, die unterhalb der Füße auf der Vorderseite die griechischsprachige Stifterinschrift und an der rechten Hockerseite die Bildhauersignatur aufweist ${ }^{39}$ (Abb. 9).

Der sog. Elgin-Thron aus Athen trägt an den Seitenwangen neuattische Reliefdarstellungen und auf der Oberkante der Rücklehne eine fragmentierte Signatur aus Individual- und Vatersname sowie Demotikon, aber offenbar ohne Verbum ${ }^{40}$. Die Inschrift war wohl nur sehr schwer zu lesen, denn die Buchstaben sind klein und bemerkenswerterweise auf die Außenkante gerichtet.

Im Sarapisbezirk zu Memphis wurde am Ende eines Dromos, der auf den Osttempel Nektanebos' II. zuführte, eine halbrunde Exedra der hellenistischen Zeit freigelegt, welche Figuren

35 M. Verzár Bass, MEFRA 102, 1990, 367 ff. Abb. 1 ff.; M. Denti, Ellenismo e romanizzazione nella X Regio (1991) 177. 179. 233. 261. 284 f. 292. 320. 336; M. L. Pagliani, Piacenza. Forma e urbanistica (1991) 33 Nr. 65; 49 Abb. 37. 38; F. Rebecchi in: Die Stadt in Oberitalien und in den nordwestlichen Provinzen des römischen Reiches, Kolloquium Köln 1989 (1991) 153 ff. Abb. 10. 11; R. Ghetti in: Atlante dei Beni Culturali dell'Emilia Romagna II (1994) 185 Abb. 3. 4; M. Verzár-Bass in: Les élites municipales de l'Italie péninsulaire des Gracques à Néron, Actes de la table ronde, Clermont-Ferrand 1991 (1996) 216 ff. Abb. 1.

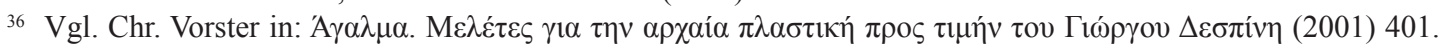

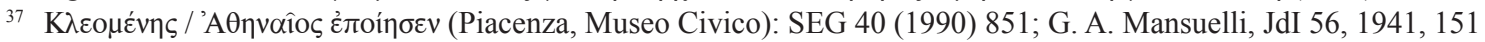
ff. Abb. 1 ff.; Richter (Anm. 11) 47 Abb. 97; G. Susini in: La statua del guerriero ferito (Ausstellungskat. Florenz 1992 [1992]) 53 Nr. 3 Abb. 9; Chr. Vorster in: KLA 415 s. v. Kleomenes III.

38 Donderer (Anm. 9) 87 f. Abb. 3. 4. 7. 8.

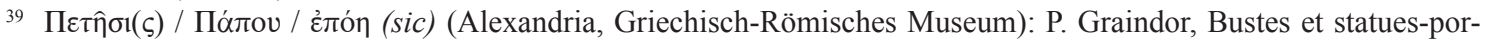
traits d'Égypte romaine (1937) 130 ff. Nr. 67 Taf. 60; L. Guerrini in: EAA VI (1965) 96 s. v. Petesis; E. Bernand, Recueil des inscriptions grecques du Fayoum I (1975) 160 f. Nr. 80 (Lit.); R. S. Bianchi in: Life in a Multi-Cultural Society, Kongreß Chicago 1990 (1992) 16 Doc B 1 Abb. 3, 2; K. Lembke, JdI 113, 1998, 113. 120. 122. 128 Nr. 17.

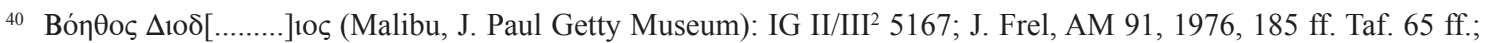
H. Meyer, Kunst und Geschichte (1983) 58 ff.; R. Vollkommer in: KLA 120 s. v. Boethos IV (Lit.). 
griechischer Dichter, Philosophen und ptolemäischer Könige(?) schmückten $^{41}$. Die Sitzstatue des Pindar trägt auf der Rückseite des Thrones eine Inschrift, von der sich der vordere Teil eines griechischen Namens erhalten hat ${ }^{42}$. Im Ausschließungsverfahren liegt eine Deutung des Namensrestes auf eine Künstlersignatur nahe. Da sich in diesem Fall die Plastik offenbar an ihrem originalen Aufstellungsort an der Flanke des Halbrundes direkt neben dem Prozessionsweg befindet, läßt sich der Anbringungsort der Inschrift leicht erklären: wer die Straße zum Tempel entlangzog, stieß als erstes auf die Pindarstatue, die er von hinten

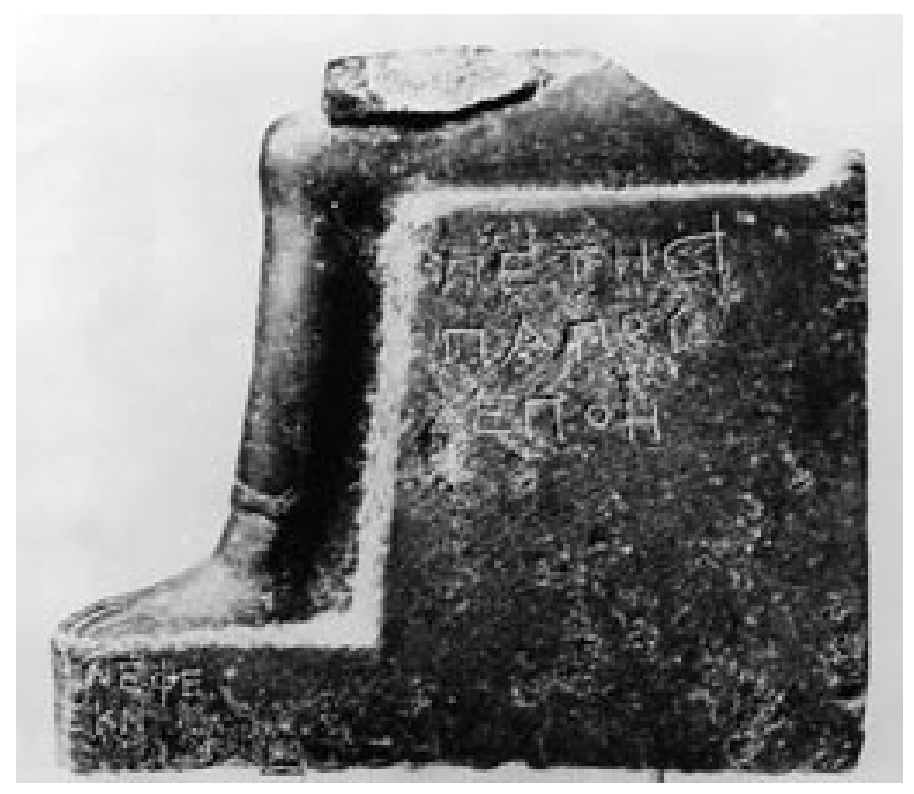

9 Sitzstatue aus dem Fayum erblickte. Auf diese Weise fiel die Künstlersignatur sofort ins Auge, was sicher intendiert war.

Wo keine Stütze, Gewandpartie oder Fläche auf einem Sitzmöbel zur Verfügung stand, war der Künstler in der Wahl des Anbringungsortes frei, wenn denn der Entschluß zur Signatur gefallen war. Jede Marmorstatue ist mit einer Plinthe gearbeitet, die für gewöhnlich in eine Basis eingelassen war. Durch Verzapfung, Verdübelung, Verklammerung oder Bleiverguß bzw. Mörtelunterlage erhielt das Werk einen besonders festen Halt. Seit dem Hellenismus nahm allerdings die Sitte zu, Plinthen partiell oder zur Gänze sichtbar zu lassen; erwiesen wird dies nicht zuletzt durch die zunehmende Anzahl von Skulpturen mit profilierten Plinthen ${ }^{43}$. Außerdem tragen immer mehr von ihnen Inschriften, sei es Signaturen, Votivtituli oder die Namen der dargestellten historischen bzw. mythischen Personen ${ }^{44}$. Zwar wird selbstverständlich die Plinthenvorderseite

41 J. Ph. Lauer - Ch. Picard, Les statues ptolemaïques du Sarapieion de Memphis (1955) bes. 148 ff. (1. Viertel 3. Jh. v. Chr.); Ch. Picard, RA 6. Serie, 47, 1956, 65 ff. 104 f.; F. Matz, Gnomon 29, 1957, 90.92 (1. Viertel 2. Jh. v.

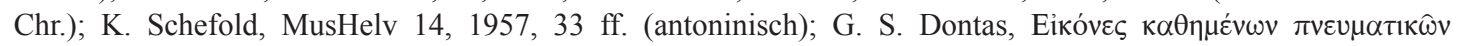

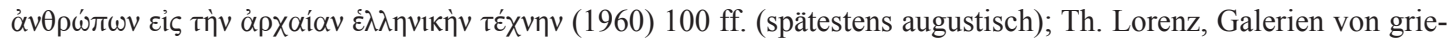
chischen Philosophen- und Dichterbildnissen bei den Römern (1965) 4 ff. 44 (kaiserzeitlich); M. Jacob-Felsch, Die Entwicklung griechischer Statuenbasen und die Aufstellung der Statuen (1969) 81. 86 Anm. 38, 2; 146 ff. Nr. 50 (1. Viertel 3. Jh. v. Chr.); H. Jucker, Gnomon 41, 1969, 79 Anm. 2 (2. Hälfte 2. Jh. v. Chr.); Fraser (Anm. 3) I 253. 255; II 402 Anm. 498; 404 Anm. 512; W. Hornbostel, Sarapis (1973) 408 ff. (1. Viertel 2. Jh. v. Chr.); M. Pietrzykowski, Rzeźby greckie z Sarapeum Memfickiego (1976); K. Gaiser, Das Philosophenmosaik in Neapel (1980) 93 Anm. 178 (antoninisch); C. Reinsberg, Studien zur hellenistischen Toreutik (1980) 118. 184 (frühes 2. Jh. v. Chr.); D. J. Thompson, Memphis under the Ptolemies (1988) 28. 116 f. (1. Hälfte 3. Jh. v. Chr.); B. S. Ridgway, Hellenistic Sculpture I (1990) 131 ff. (hellenistisch); A. Schmidt-Colinet, HASB 14, 1991, 58 ff.; A. Lewerentz, Stehende männliche Gewandstatuen im Hellenismus (1993) 167 ff. (2. Jh. v. Chr.); G. Hölbl, Geschichte des Ptolemäerreiches (1994) 256 f. (70er Jahre des 1. Jhs. v. Chr.); S. von Thüngen, Die frei stehende griechische Exedra (1994) 62 f. (1. Viertel 3. Jh. v. Chr.); P. Zanker, Die Maske des Sokrates (1995) 166 f. (spätes 3./frühes 2. Jh. v. Chr.); K. Schefold, Die Bildnisse der antiken Dichter, Redner und Denker (1997) 358. 535.560 f. Anm. 109 (antoninisch); H. von Steuben in: Hellenistische Gruppen. Gedenkschrift A. Linfert (1999) 62 f. (1. Viertel 3. Jh. v. Chr.).

${ }^{42} \Delta$ tovvol[.....] (in situ): O. Brendel, RM 51, 1936, 47; J. und L. Robert, Bulletin Epigraphique 1953, 236; J. Marcadé, REA 58, 1956, 123 Anm. 1; Fraser (Anm. 3) I 255; II 404 Anm. 512; Pietrzykowski (Anm. 41) 10 ff. Abb. 6-10; B. Tkaczow, EtTrav 13, 1983, 399; Donderer (Anm. 9) 94; R. Vollkommer in: KLA 174 s. v. Dionysi(....)(Lit.).

${ }^{43}$ U. Kron, JdI 92, 1977, 148 ff. bes. $160 \mathrm{ff}$.

44 Kron (Anm. 43) $151 \mathrm{ff}$. 


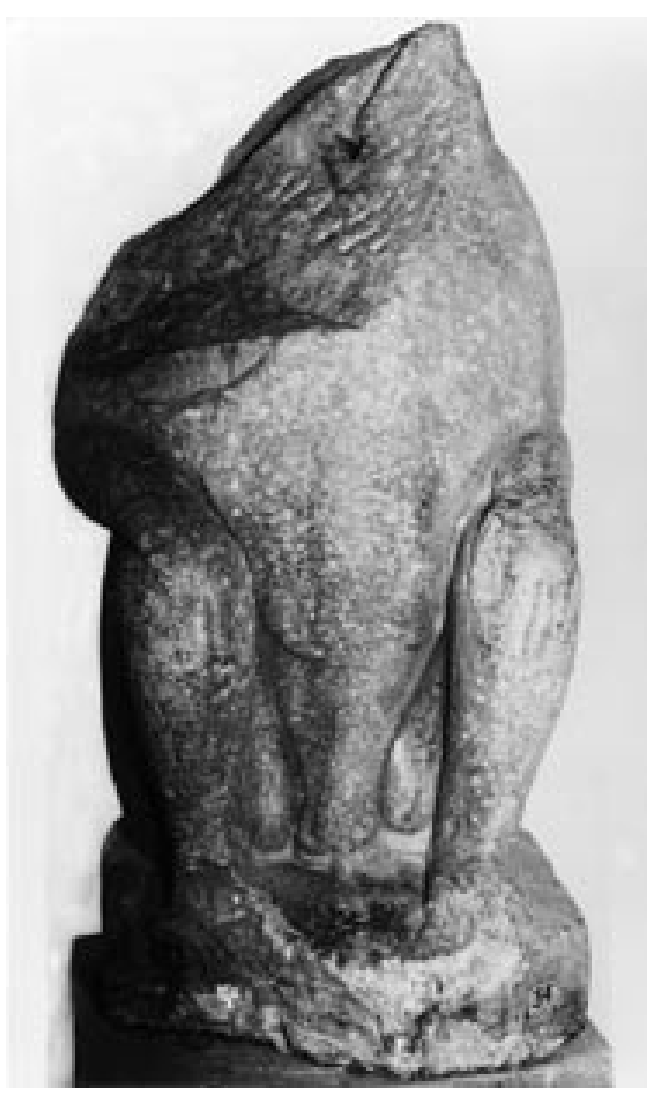

10 Hundsköpfige Granitstatue aus Rom

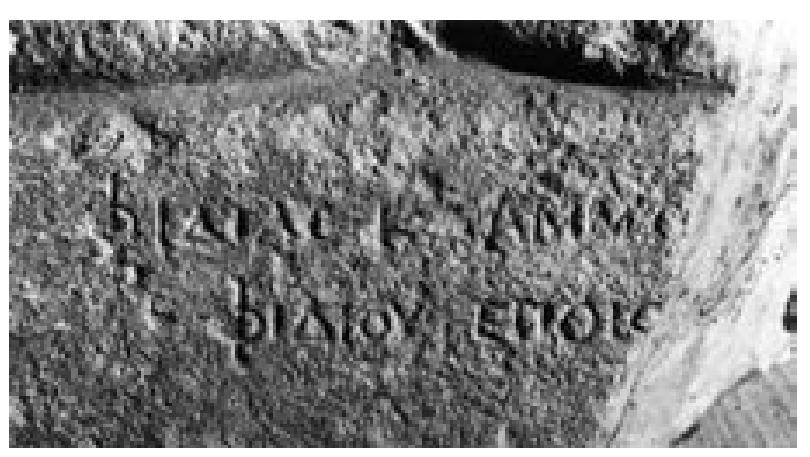

11 Signatur des Phidias und des Ammonios

bevorzugt, doch erscheinen die Namen Porträtierter gelegentlich auf der rechten Nebenseite ${ }^{45}$. Dies trifft auch für einige wenige Künstlernennungen zu.

Eine Doppelsignatur findet sich auf der rechten Plinthennebenseite einer hundsköpfigen Granitstatue ägyptischen Typs aus dem Iseum Campense in Rom $^{46}$ (Abb. 10. 11). Vorder- und linke Nebenseite trugen ehedem die griechische bzw. lateinische Stifterinschrift, letzte erweitert durch die Consulsangabe, so daß dadurch die Weihung und wohl auch annähernd die Herstellung auf das Jahr 159 n. Chr. festgelegt $\operatorname{sind}^{47}$.

Ebenfalls von der Dedikationsinschrift wird die Plinthenvorderseite einer frühkaiserzeitlichen(?) Sitzstatuette des Herakles Epitrapezios aus Ninive eingenommen (Abb. 12); der Name des Bildhauers erscheint wiederum auf der Nebenseite, doch diesmal ausnahmsweise auf der linken ${ }^{48}$ (Abb. 13). Wie schon bei dem zuvor genannten Monument liegt, wie nicht anders zu erwarten, das Hauptgewicht auf der Nennung des Auftraggebers, der Künstlername ist dagegen zweitrangig; diese Wertung spiegelt sich eindeutig in der Wahl des Anbringungsortes. Vor allem auf Basen früherer Zeit wurde dasselbe Faktum oft durch die Entscheidung für einen kleineren Schrifttypus bei der Signatur unterhalb der Dedikationsinschrift zum Ausdruck gebracht.

Regelgerecht für die rechte Plinthenseite hat sich der Bildhauer der möglicherweise hadrianischen Kultstatue des Zeus Ägiochos aus dem Kapitol von Kyrene als Ort für die Signatur entschieden $^{49}$. In ähnlicher Weise wurde für die Frauenstatue (Agrippina d. J.?) aus dem Kaiser-

45 Kron (Anm. 43) 155 ff.

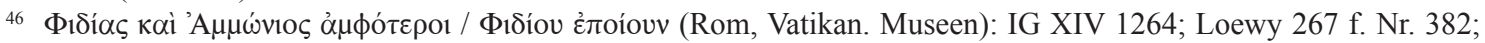
A. Roullet, The Egyptian and Egyptianizing Monuments of Imperial Rome (1972) 125 Nr. 245 (Lit.) Abb. 251-253; J.-Cl. Grenier, Museo Gregoriano Egizio (1993) 39 f. Nr. IV 6 Taf. 11; K. Lembke, Das Iseum Campense in Rom (1994) 142 f. Nr. 8; 238 Nr. E 36 Taf. 43, 1. 2; E. Paul - W. Müller in: KLA 31 f. s. v. Ammonios (Lit.).

47 Moretti (Anm. 23) 82 f. Nr. 1588.

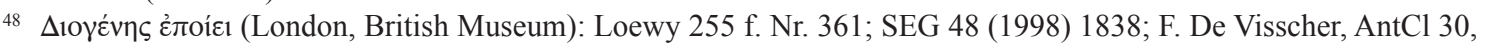
1961, 124 Taf. 19 Abb. 14; E. Bartman, Ancient Sculptural Copies in Miniature (1992) 160. 163.181 Nr. 15 (Lit.) Abb. 90. 91; A. Latini, RdA 19, 1995, 65 Abb. 12; J. E. Reade, Iraq 60, 1998, 69 f. Nr. 4 Abb. 4; A. Herr in: KLA 172 s. v. Diogenes II (Lit.); P.-L. Gatier, Bulletin Epigraphique 2002, 454.

49 Znvíwv Znvíwvos (Kyrene, Museum): SEG 20 (1964) 726; P. E. Arias, RIA 9, 1942, 98 ff. Abb. 1. 2 Taf. 1; F. Chamoux, BCH 70, 1946, 69 ff. Taf. 4; Richter (Anm. 11) 47. 52 Abb. 91; P. Moreno in: EAA VII (1966) 1248 s. v. Zenion Abb. 1384; C. Parisi Presicce in: L'Egitto in Italia dall'antichità al medioevo. Atti del III. Congresso Internazionale Italo-Egiziano, Rom-Pompeji 1995 (1998) 477 Abb. 4; S. Ensoli, QuadALibya 18, 2003, 65 f. 81 Abb. 21. 34. 35. 




12 Herakles aus Ninive

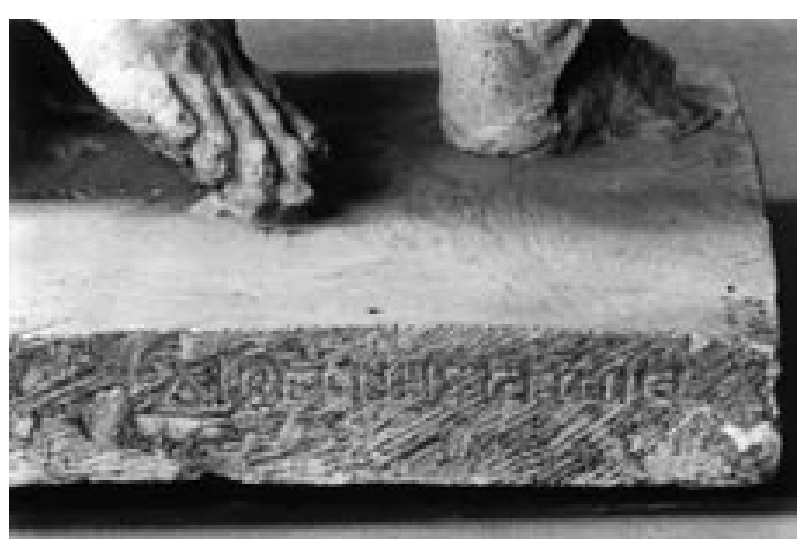

13 Signatur des Diogenes

kulttempel im vormaligen Metroon von Olympia die rechte Plinthennebenseite gewählt ${ }^{50}$ (Abb. 14. 15). Allerdings ist in diesem Fall nicht zu entscheiden, ob die rechteckige Plinthe bei der antiken Aufstellung überhaupt sichtbar war, denn die übrigen Statuen desselben Fundortes weisen Plinthen unregelmäßiger Form mit ungeglätteten Seitenflächen auf ${ }^{51}$, waren also wohl in die sicher zu postulierenden Basen eingelassen. Aber selbst wenn die Plinthe der Frauenstatue auf dem Basisblock gestanden haben sollte, war die Inschrift kaum lesbar: nach Aussage der Fundsituation des gesamten Ensembles stand die Statue nämlich vor der Nordwand der Cella, so daß die rechte Nebenseite mit der Signatur dem im Westen gelegenen Eingang abgewandt ${ }^{52}$ und auf diese Weise immer verschattet war.

Im Sepulkralbereich begegnen des öfteren Inschriften, die nicht unbedingt gelesen werden sollten $^{53}$. Aber selbst unter den klassischen Grabsteinen Thessaliens mit Angabe des Grabinhabers erscheint dessen Name nicht - wie eigentlich zu erwarten wäre - auf der Front-, sondern auf der Nebenseite ${ }^{54}$. Auf der Rückseite eines spätklassischen Grabreliefs(?) von der Agora in Kyrene befindet sich ein Monogramm, das nach Aussage von Analogien am ehesten als Bildhauerzeichen zu deuten ist ${ }^{55}$. Ein aufs Jahr genau datierter ${ }^{56}$ Sarkophag aus Sidon trägt den Namen des Verstorbenen und den des Handwerkers auf der Nebenseite ${ }^{57}$ (Abb. 16); möglicherweise war der

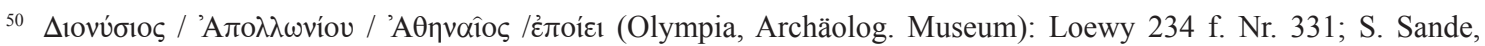
ActaAArtHist 5, 1985, 158 Abb. 3; K. Hitzl, Die kaiserzeitliche Statuenausstattung des Metroon, OF XIX (1991) 43 ff. Nr. 3 (Lit.) Taf. 14c; 15-19; 39b; 40c; ders. in: KLA 179 s. v. Dionysios XI (Lit.); D. Boschung, Gens Augusta (2002) 101 Nr. 33, 5 Taf. 81, 2.

51 Hitzl (Anm. 50:1991) passim.

52 Hitzl (Anm. 50:1991) 25 ff. 101 ff. Abb. 5. 8.

53 Donderer (Anm. 5) $108 \mathrm{f}$.

54 H. Biesantz, Die thessalischen Grabreliefs (1965) Nr. 8. 14. 20a. 27 Taf.

55 Kyrene, Archäolog. Museum: M. Donderer, Boreas 23/24, 2000/01, 90 (Lit.) Taf. 9 Abb. 23. 24; E. Fabbricotti in: N. Bonacasa - S. Ensoli, Cirene (2000) 197 Abb.

56 Allerdings ist nicht ganz sicher, auf welche Ära sich die Zahl 292 bezieht: Die seleukidische Ära ist auszuscheiden, da dies dem Jahr 20 v. Chr. entsprechen würde, zu früh für die Art des Sarkophags. Die Alternative besteht zwischen der Ära von Sidon (= 181 n. Chr.) und derjenigen von Antiochia (= 244 n. Chr.).

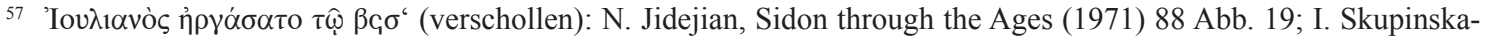
Løvset in: Ancient Portraiture. Image and Message (1992) 226 f. Abb. 4. 


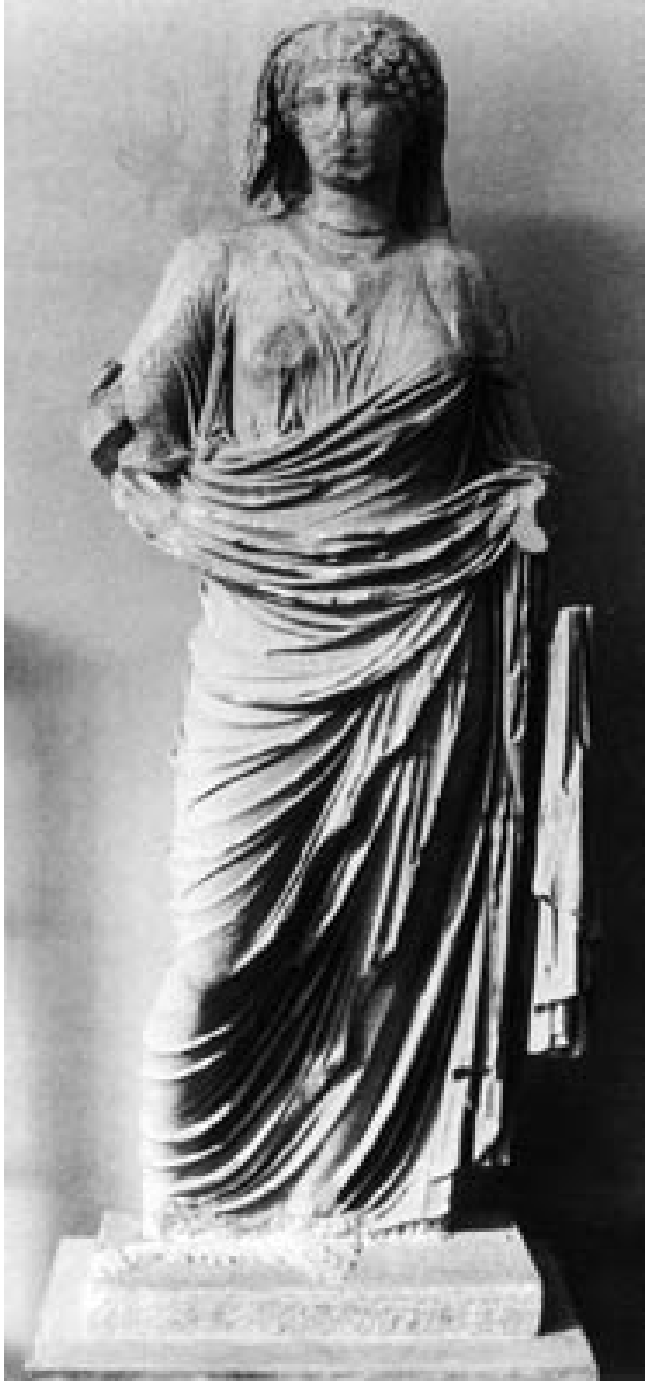

14 Frauenstatue aus Olympia

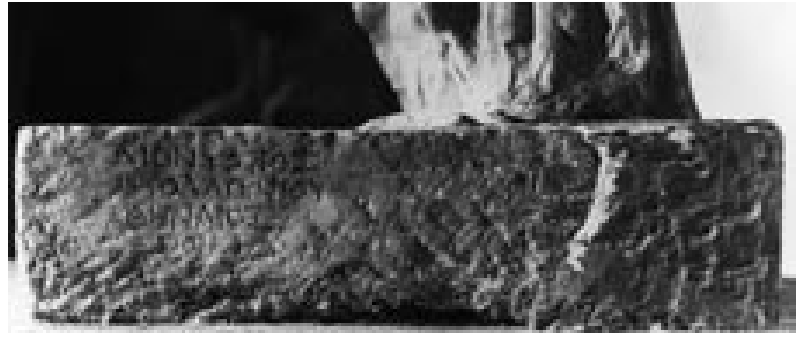

15 Signatur des Dionysios

Anbringungsort jedoch durch die ehemalige Aufstellungssituation bedingt, die uns heute nicht mehr bekannt ist. Dieselbe Erklärung trifft vielleicht auch für einen vierseitig ausgearbeiteten Girlandensarkophag (Abb. 17) der zweiten Hälfte des 2. Jahrhunderts n. Chr. aus Antiochia/Orontes zu, den der Bildhauer auf der Schmalseite signiert hat ${ }^{58}$ (Abb. 18). Die Nennung des Sarkophaginhabers befand sich entweder nur in Farbe auf der Wandung aufgemalt oder war am Grabbau angebracht, der den Sarkophag barg.

Aus derselben Region stammt ein etwa gleichzeitig gearbeiteter Klinensarkophag, der auf der untersten Basisprofilleiste der Rückseite einen Namen im Genetiv trägt ${ }^{59}$. Da Namensangaben an dieser Stelle in der Regel auf Bildhauer weisen ${ }^{60}$, dürfte dies auch im vorliegenden Fall zutreffen, wenngleich ein Bezug zum Auftraggeber nicht völlig auszuschließen ist ${ }^{61}$.

Signaturen erscheinen zudem auf der rechten Nebenseite einer Ostothek aus Bayyurdu-Sobran (Isaurien) $^{62}$ und auf der linken Nebenseite eines Grabaltars, der in der Nähe von Alanya gefunden wurde $^{63}$ (Abb. 19. 20); beide Monumente lassen sich nur grob in die fortgeschrittene Kaiserzeit datieren. Nördlich von Kütahya wurde ein Votiv-

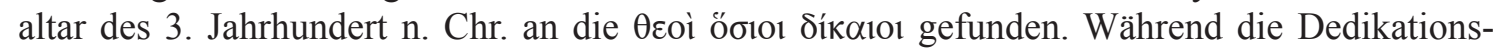

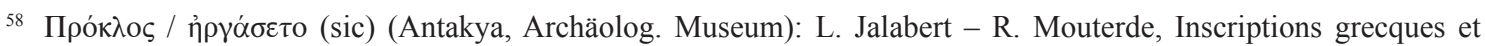
latines de la Syrie III 2 (1953) 531 Nr. 989; N. Himmelmann, Sarkophage in Antakya (1970) 8 f. Taf. 6. 7; J. und L. Robert, Bulletin Epigraphique 1973, 57; G. Koch - H. Sichtermann, Römische Sarkophage (1982) 435 Anm. 1; 473. 568.

$59 \Phi \lambda(\alpha$ ovíov) Kúpov (Antakya, Archäolog. Museum): Himmelmann (Anm. 58$) 15$ ff. Abb. 12. 13. Taf. 11-14; Koch - Sichtermann (Anm. 58) 369 Anm. 4; 372 Anm. 62; 381. 425. 431 Nr. 37; 443 ff. 458 f. Abb. 409. 410. 456; G. Koch, BJb 189, 1989, 187 Abb. 32; S. Rogge in: Grabeskunst der römischen Kaiserzeit (1993) 114 f. 120 ff. Taf. 53.

${ }^{60}$ Donderer (Anm. 55) 83.

${ }_{61}$ Vgl. G. Koch, Sarkophage der römischen Kaiserzeit (1993) 47 mit Anm. 229.

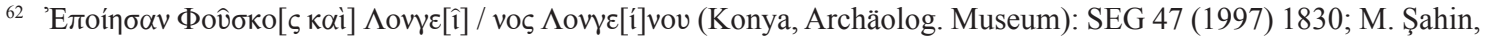
EpigrAnat 29, 1997, 78 f. Nr. 3 Taf. 2. 3; Cl. Brixhe, Bulletin Epigraphique 1999, 547.

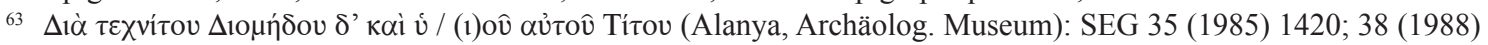
1487; J. Nollé - S. Şahin - Ch. Vorster, EpigrAnat 5, 1985, 132 ff. Nr. 10 Taf. 20; St. Hagel - K. Tomaschitz, Repertorium der westkilikischen Inschriften (1998) 169 Nr. 19; T. Lochman in: KLA 173 f. s. v. Diomedes I. 
inschrift oberhalb des Reliefs der Schaftvorderseite zu lesen ist (Abb. 21), wurde die Signatur der beiden Bildhauer auf den nicht voll ausgearbeiteten oberen Abschlußprofilleisten der Rückseite eingemeißelt ${ }^{64}$ (Abb. 22).

Nicht für die gesamte Meißelarbeit, sondern offensichtlich nur für den Text zeichnete ein Handwerker auf der oberen Profilleiste der rechten Nebenseite eines Sarkophags aus der Nähe Roms verantwortlich, welcher durch die Angabe der Consuln ins Jahr 217 n. Chr. datiert ist ${ }^{65}$. Informationen zum Grabinhaber befinden sich auf der von zwei Eroten gehaltenen tabula ansata auf der Frontseite. Allerdings ist der Titulus sekundär, da er auf dem nachträglich vertieften Tafelgrund erscheint und somit einen älteren eradierten Text ersetzte $^{66}$. Daß die Inschrift der Nebenseite gleichzeitig mit dem sekundären Titulus der Vorderseite ist, beweist die erneute Nennung des Verstorbenen. Zwar unterscheiden sich beide Texte im Schriftduktus, doch ist dies wohl dadurch zu erklären, daß die Buchstaben der Vorderseite kalligraphisch komponiert sind, die der Nebenseite aber

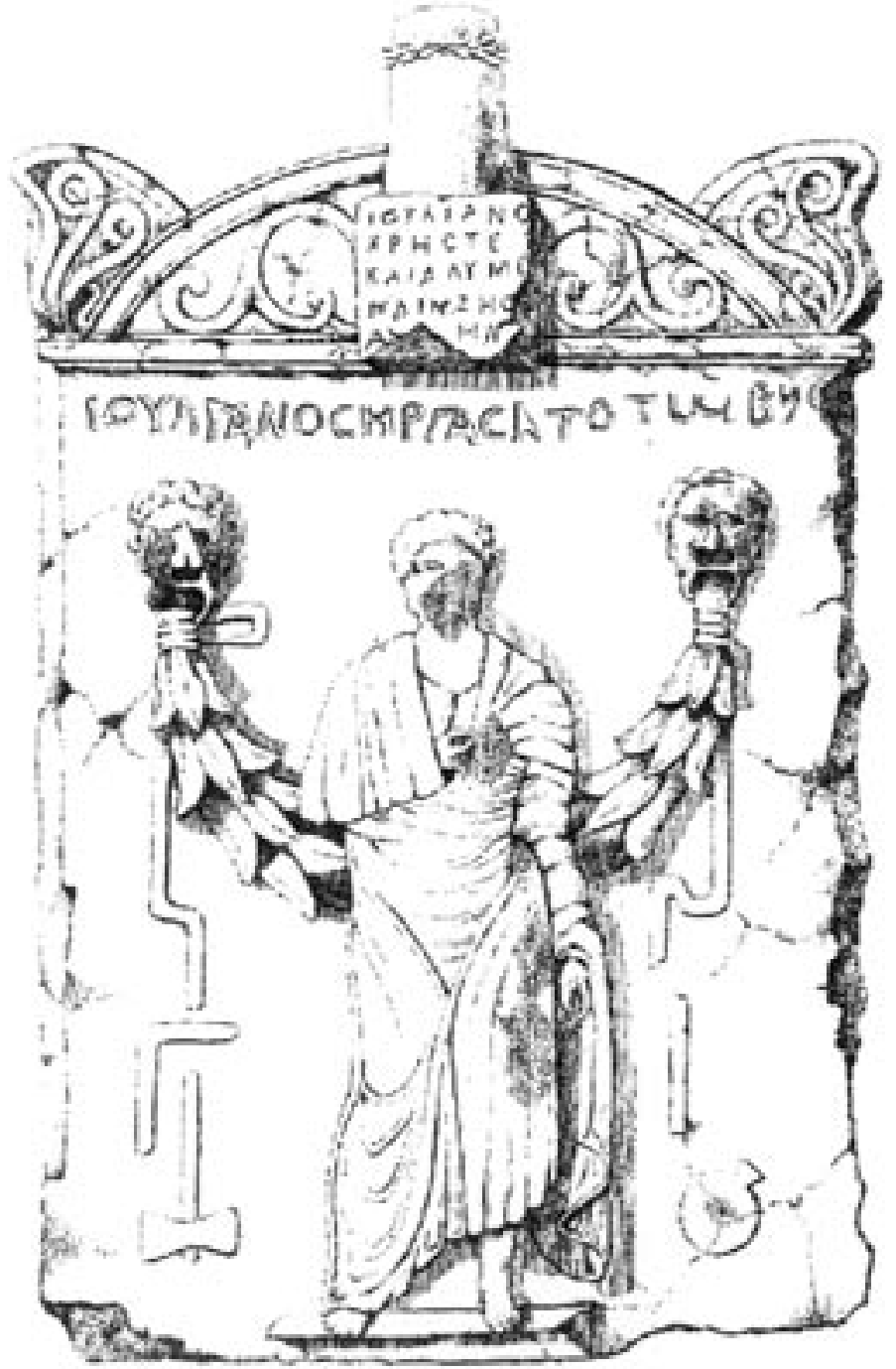

16 Sarkophag aus Sidon nicht.

Die große Mainzer Iuppitersäule ${ }^{67}$, wohl anläßlich eines gescheiterten Attentatsversuches auf Kaiser Nero errichtet, birgt die Widmungsinschrift innerhalb des profilgerahmten oberen Sockels, während wiederum auf der rechten Nebenseite das obere Abschlußgesims des unteren Sockels die Signatur trägt ${ }^{68}$, die in dieser Monumentgattung bis heute singulär ist ${ }^{69}$.

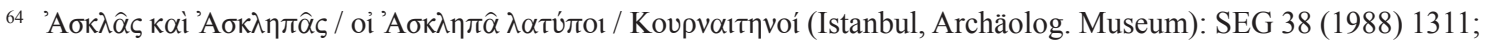
Loewy 272 Nr. 391; E. Gibson, The 'Christians for Christians' Inscriptions of Phrygia (1978) 69 Nr. 14; M. Ricl, EpigrAnat 18, 1991, 14 f. Nr. 26 (Lit.) Taf. 5; T. Lochman in: KLA 98 s. v. Asklas (Lit.).

65 Scripsit Ampelius libertus (Rom, bei Villa Borghese): CIL VI 8498; ILS 1738; ILCV 3332; G. Bovini, I sarcofagi paleocristiani (1949) 14 f. Nr. 1 (Lit.); L. Quilici, Collatia (1974) 708 Nr. 627 Abb. 1586-1588; Koch - Sichtermann (Anm. 58) 273 Anm. 13; E. Equini Schneider, La 'Tomba di Nerone' sulla Via Cassia (1984) 68 f. Taf. 11b. 12; G. Koch, Frühchristliche Sarkophage (2000) 4. 7. 54. 101 f. 123. 227.238 (Lit.); 355. 444 f. Abb. 13.

${ }_{66}$ G. Bovini - H. Brandenburg, Repertorium der christlich-antiken Sarkophage I (1967) 387 Nr. 929 (Lit.) Taf. 148.

${ }^{67}$ G. Bauchhenß, Die große Iuppitersäule aus Mainz (1984) mit Lit.

68 Samus et Severus Venicari f(ilii) sculpserunt (Mainz, Mittelrheinisches Landesmuseum): CIL XIII 11806; ILS 9235; P. Moreno in: EAA VI (1965) 1107 s. v. Samus; VII (1966) 230 s. v. Severus; G. Rupprecht in: Die Römer in Rheinland-Pfalz (1990) 464 f. Abb. 84.

${ }^{69}$ G. Bauchhenß - P. Noelke, Die Iupitersäulen in den germanischen Provinzen (1981) bes. 272 ff. (Lit.). 


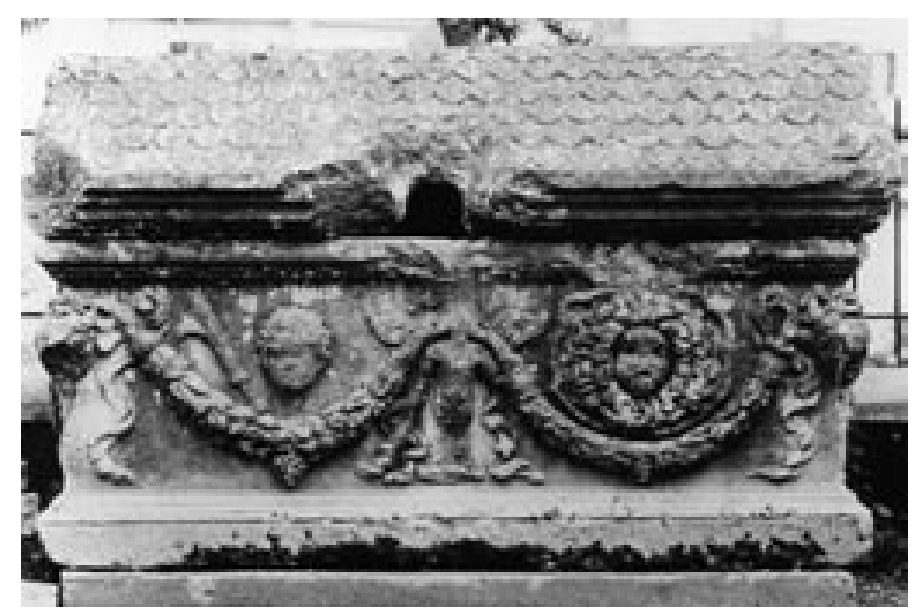

17 Girlandensarkophag aus Antiochia/Orontes

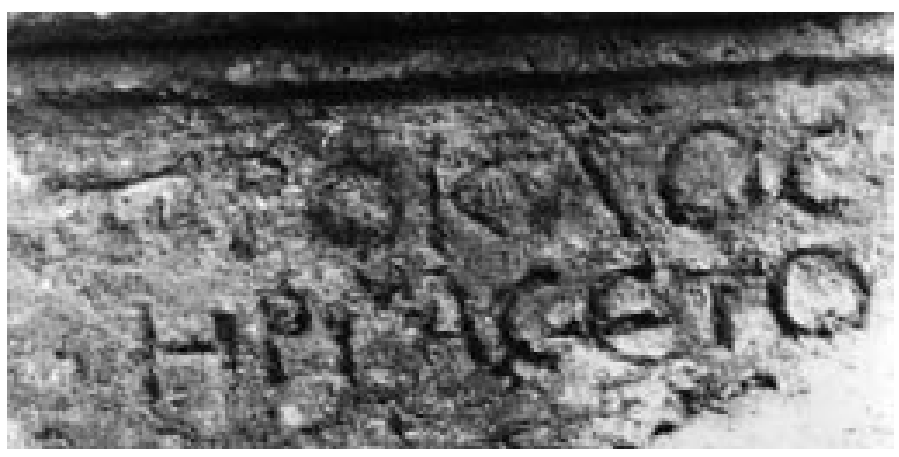

18 Signatur des Proklos
Einen Sonderfall stellt der vor der toskanischen Küste im Meer entdeckte sog. Apoll von Piombino dar (Abb. 23). Die Legierung der Bronze, stilistische Beobachtungen $^{70}$ und die Form der Votivinschrift auf dem linken Fuß der Statue führten zu dem Ergebnis, daß es sich nicht um eine archaische Skulptur handelt, wie die Forschung lange annahm ${ }^{71}$, sondern um ein archaistisches Werk des 1. Jahrhunderts v. $\mathrm{Chr}^{72}$. Einzigartig sind Träger und Fundort der Signatur: ein Bleistreifen im Inneren der Statue, auf dem zwei Künstler genannt waren ${ }^{73}$ (Abb. 24). Die einzig überzeugende Erklärung für diese Art der Deponierung: Die Skulptur wurde in der Antike zwar als Fälschung konzipiert, doch wollten die Autoren in ihrem Stolz nicht auf eine Signatur verzichten; diese konnte jedoch nicht offen, sondern nur versteckt angebracht werden. Ähnliche Fälle sind übrigens aus der Neuzeit bekannt ${ }^{74}$.

Zusammenfassend läßt sich

feststellen, daß eine Reihe plastischer Werke die Signatur nicht, wie zu erwarten wäre, an der Hauptansichtsseite, sondern entweder auf der Neben- oder gar auf der Rückseite trägt ${ }^{75}$. Be-

70 Vgl. jetzt auch eine ähnliche Bronzestatue aus Pompeji, Haus des Polybios (IX 13, 1-3): C. C. Mattusch, Classical Bronzes (1996) 139 f. Farbtaf. 5; dies. in: Regional Schools in Hellenistic Sculpture. Proceedings of an International Conference, Athens 1996 (1998) 152 f. Abb. 6; B. S. Ridgway, Hellenistic Sculpture III (2002) 148 Taf. 59; V. M. Strocka, JdI 117, 2002, 114 (Lit.) Abb. 38. 39; L. Fergola in: Storie da un'eruzione. Pompei, Ercolano, Oplontis (Ausstellungskat. Neapel 2003 [2003]) 424 Nr. IV 755 Abb.

71 G. M. A. Richter, Kouroi (1960) 144 f. Nr. 181 (Lit.) Abb. 533-540; zuletzt noch D. Kreikenbom in: Polyklet. Der Bildhauer der griechischen Klassik (Ausstellungskat. Frankfurt/M. 1990/91 [1990]) 38; vgl. auch die Lit. bei Fuchs (Anm. 16) 23 mit Anm. 1. 3-6.

72 B. S. Ridgway in: AntPl VII (1967) 43 ff. Abb. 1. 4-11 Taf. 24-34; dies., Roman Copies of Greek Sculpture (1984) 22 f.; M. A. Zagdoun, La sculpture archaïsante dans l'art hellénistique et dans l'art romain du Haut-Empire (1989) 147 f. 213. 247 Nr. 347; Cl. Rolley, La sculpture grecque I (1994) 399; B. S. Ridgway, The Archaic Style in Greek Sculpture $^{2}$ (1993) 458 Abb. 151; Mattusch (Anm. 70:1996) 139. 225 Taf. 6; B. S. Ridgway in: Mattusch (Anm. 30) 129 Abb. 7; Mattusch (Anm. 70:1998) 151 f. Abb. 5; Fuchs (Anm. 16) 23 ff. (Lit.) 40 f. Taf. 24. 25; Ridgway (Anm. 70) 147 f. Taf. 58a-c; M.-A. Zagdoun in: From the Parts to the Whole II. Acta of the 13th International Bronze Congress, Cambridge, Mass. 1996 (2002) 66 f. Abb. 1.

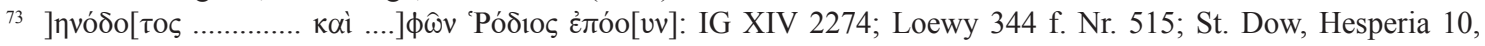
1941, 357 ff. Abb.; Ridgway (Anm. 72:1967) 44. 68 Abb. 1; V. C. Goodlett, Collaboration in Greek Sculpture. The Literary and Epigraphical Evidence (Diss. New York 1989) 153 f. 198; dies., AJA 95, 1991, 677 f.; Fuchs (Anm. 16). 23 Abb. 1.

74 Fuchs (Anm. 16) 40 f. mit Anm. 17.

75 Hingewiesen sei in diesem Zusammenhang auch auf eine Bronzestatuette aus Carnuntum, die eine stark abgekürzte Signatur des Produzenten auf der Unterseite trägt: Donderer (Anm. 55) 89 Taf. 9 Abb. 20. 21; ders., Laverna 14, 2003, 109 f. (Lit.). 

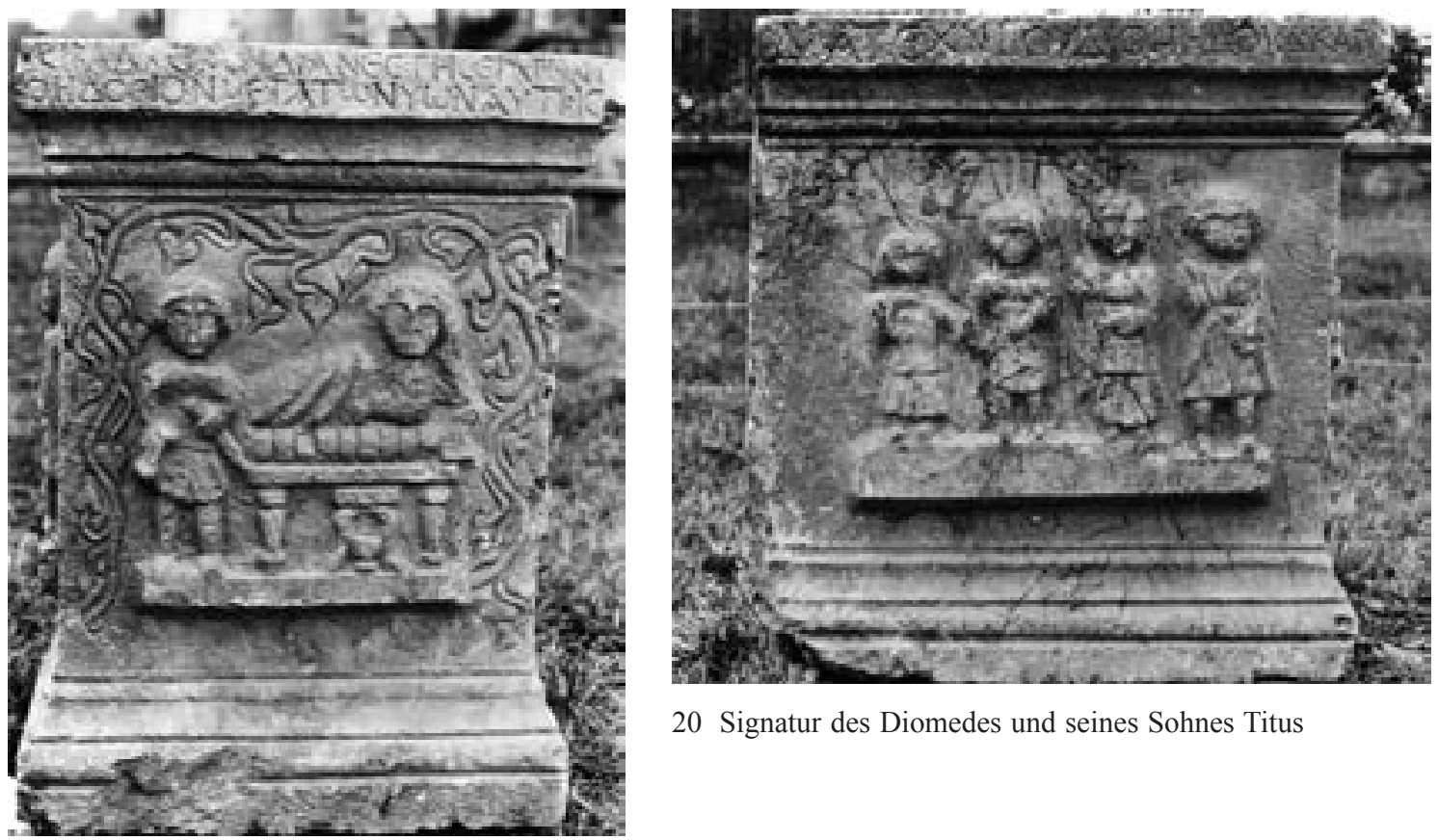

20 Signatur des Diomedes und seines Sohnes Titus

19 Grabaltar aus der Umgebung von Alanya
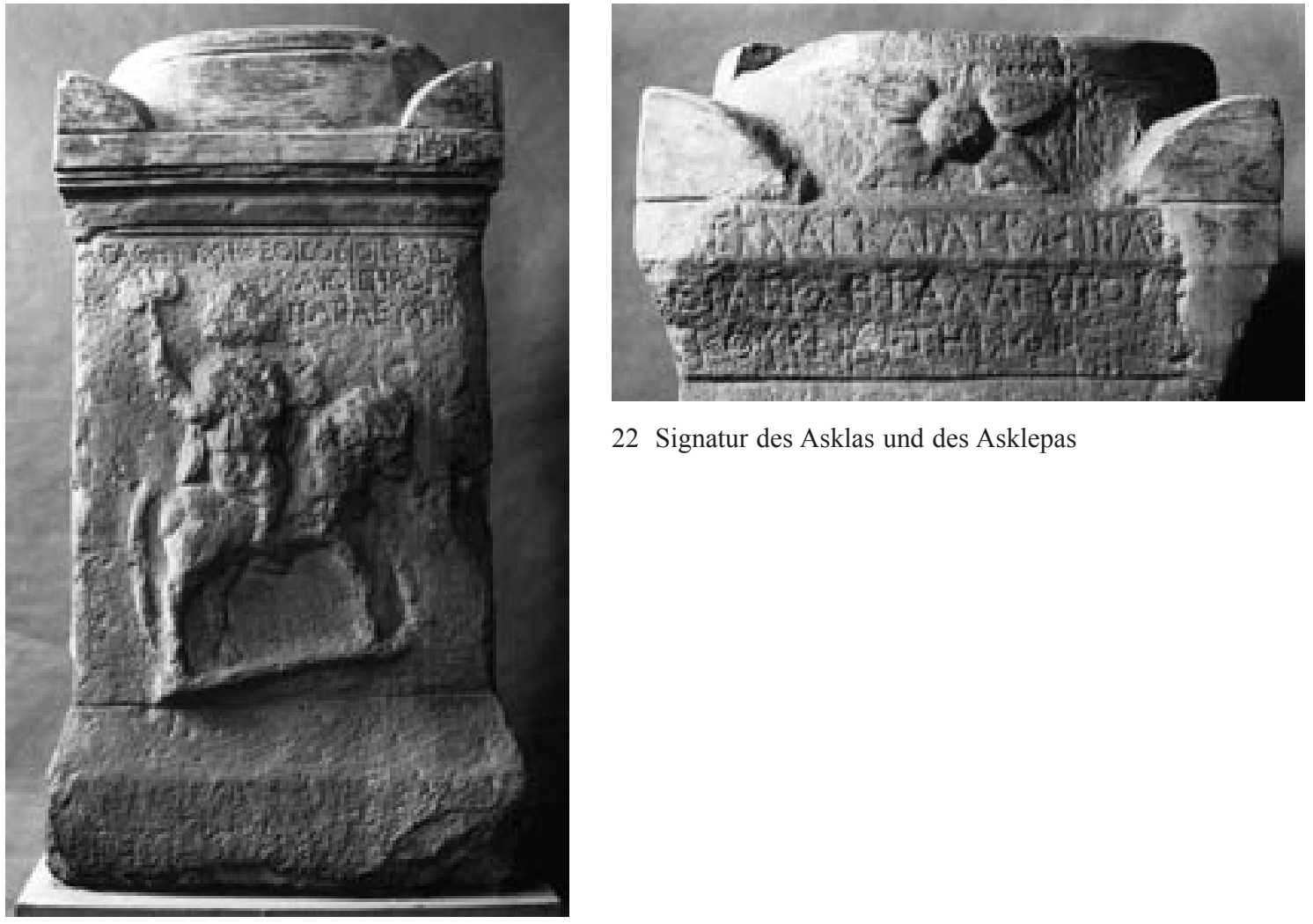

22 Signatur des Asklas und des Asklepas

21 Votivaltar aus der Umgebung von Kütahya 




merkenswert ist zudem, daß dort, wo dem Bildhauer eine Wahlmöglichkeit gegeben war, z. B. bei Plintheninschriften, die rechte Nebenseite deutlich bevorzugt wurde ${ }^{76}$. Dies hat wohl zwei Gründe: Zum ersten ließ sich der Text leichter plazieren, indem er an der linken vorderen Plinthenecke beginnen konnte. Zweitens ist es für Lebewesen offenbar ein Naturphänomen, sich instinktiv gegen den Uhrzeigersinn zu bewegen. In der Neuzeit beweisen dies nicht nur die gelenkte Bewegungsführung in Einkaufszentren sowie die Rennen jeder nur denkbaren Art auf festgelegten Strecken und in Stadien. Auch im Altertum verliefen die Wagenrennen im Zirkus in dieser Richtung - wie zahlreiche Abbildungen auf Reliefs und Mosaiken zeigen ${ }^{77}$. Zudem ist gerade dann mit einer Signierung an nicht (sofort) sichtbarer Stelle zu rechnen, wenn der Name zusätzlich durch Abkürzung oder Monogrammform verfremdet ist $^{78}$. Andererseits fällt auf, daß bei rundplastischen Votiven die Nennung des Stifters erwartungsgemäß auf der Hauptseite erfolgt, eine eventuell vorhandene Künstlerinschrift aber nur äußerst selten direkt angeschlossen wird. In der Regel ist sie auf der rechten Nebenseite angebracht $^{79}$.

Nach diesen Beobachtungen ist davon auszugehen, daß ein Monument, wenn überhaupt, in der Antike gegen den Uhrzeigersinn umschritten wurde und der Betrachter sich in einigen Fällen sogleich für seine Bereitschaft mit der zusätzlichen Information der Künstlernennung belohnt sah. So verlangte die Darstellung einer nackten Aphrodite geradezu die Begut-

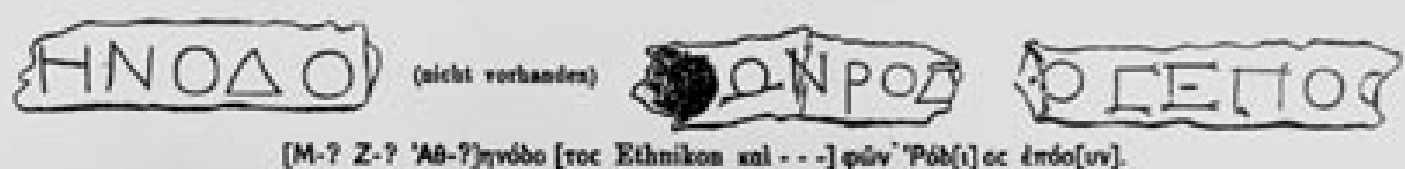

24 Signatur auf Bleistreifen

76 o. Anm. 46. 49. 50. - Vgl. auch Benennungen der dargestellten Personen auf der rechten Plinthennebenseite: Kron (Anm. 43) $155 \mathrm{ff}$.

77 M. Floriani Squarciapino, RendLinc, 8. Serie, 34, 1980, 284 ff. Taf. 1. 2; J. H. Humphrey, Roman Circuses (1986) 176 ff. Abb. 78. 80. 83. 95-103. 107-120; A. Hönle - A. Henze, Römische Amphitheater und Stadien (1981) $93 \mathrm{ff}$. Abb. $58 \mathrm{ff}$.

78 Vgl. die Belege bei Donderer (Anm. 55) 79 ff. mit Anm. 38. 41. 51-60 (vgl. jetzt dazu F. Heger, Archeologia Warszawa 53, 2002, 151 f.); 62. 67. 111. 150. 154.

79 o. Anm. 38. 39. 76. 
achtung der Rückansicht, wie wir durch Schriftquellen zur Aphrodite des Praxiteles in Knidos wissen $^{80}$.

Ein Sarkophag konnte im Grabbezirk oder im Grabbau so aufgestellt sein, daß der Blick des Eintretenden zunächst auf die Schmalseite mit der Signatur fiel, die Nebenseite dadurch zumindest für den Produzenten aufgewertet wurde ${ }^{81}$. In vergleichbarer Weise ließe sich vielleicht manche Signatur auf der Rückseite von Statuen verstehen, doch hat sich in der Regel der primäre Aufstellungsort nicht erhalten. Allerdings gibt es immerhin eine Ausnahme: den sitzenden Pindar aus einer Exedra im Sarapis-Heiligtum von Memphis ${ }^{82}$.

Ähnliches gilt auch für Kultstatuen, an denen für gewöhnlich keine Signaturen zu finden sind, zumal die zugehörige Basis in der Regel keine Stifterinschrift trägt, an welche die Künstlernennung hätte angehängt werden können ${ }^{83}$. Bei den wenigen Ausnahmen erscheinen die Signaturen entweder an der Nebenseite ${ }^{84}$ oder gar an versteckter Stelle ${ }^{85}$.

Wir können davon ausgehen, daß sich in der Antike zwei Antipoden gegenüberstanden: Auf der einen Seite der zahlende Auftraggeber, der - zumindest in römischer Zeit - gerade bei Nennung seines Namens den eigenen Ruhm in der Regel nicht durch eine Künstlersignatur geschmälert sehen wollte, auf der anderen Seite der Bildhauer, der seine Wirkung auf die Umund Nachwelt im Auge hatte ${ }^{86}$; so muß wohl auch die Signatur im Inneren des sog. Apoll von Piombino verstanden werden ${ }^{87}$.

Wahrscheinlich hat der Auftraggeber in den meisten Fällen dem Künstler eine Signierung untersagt und nur in Ausnahmefällen auf der Vorderseite genehmigt. Vielleicht als Kompromiß ist ein Teil derjenigen Signaturen anzusehen, die an abgelegener oder versteckter Stelle plaziert sind. Massenware, die nicht im Auftrag, sondern auf Vorrat hergestellt wurde, brauchte betreffs Signaturen auf die Wünsche des Bestellers keine Rücksicht zu nehmen. Allerdings begegnen bei griechischen $V_{a s e n}{ }^{88}$ und Reliefbechern ${ }^{89}$ sowie bei römischen Gläsern ${ }^{90}$ gelegentlich Ausnahmen, indem Signaturen auf der Gefäßunterseite erscheinen ${ }^{91}$. Bei hellenistischen $^{92}$ und römischen ${ }^{93}$ Terrakotten sowie römischen Tonlampen ${ }^{94}$ wurde die Rückseite bzw. die

80 o. Anm. 28.

81 o. Anm. 57. 58.

82 o. Anm. 42.

83 D. Damaskos, Untersuchungen zu hellenistischen Kultbildern (1999) 219 ff.

84 o. Anm. 49. 50

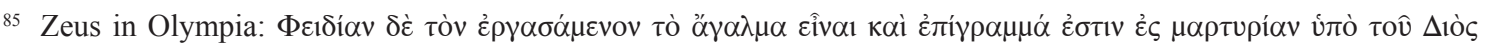

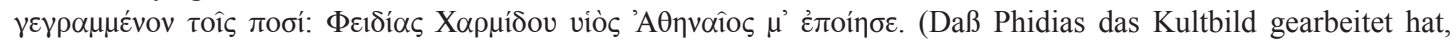
dafür dient als Beweis auch eine Inschrift, die unter den Füßen des Zeus geschrieben ist: Phidias, Sohn des Charmides, aus Athen hat mich geschaffen.): Paus. 5, 10, 2 = Overbeck, Schriftquellen Nr. 692. 618; K. D. S. Lapatin, Chryselephantine Statuary in the Ancient Mediterranean World (2001) 175. - Vgl. neuerdings zur Zeus-Statue allgemein St. Vlizos, Der thronende Zeus (1999) 5 ff.; Lapatin a. O. 79 ff. jeweils mit Lit.

86 Cic. Tusc. 1, 34. - Vgl. auch F. Kudlien, Die Rolle der Konkurrenz im antiken Geschäftsleben, Münstersche Beiträge zur antiken Handelsgeschichte 13, 1, 1994, $1 \mathrm{ff}$.

87 o. Anm. 73.

88 J. Neils in: Essays in Honor of Dietrich von Bothmer I (2002) $240 \mathrm{f}$.

89 G. Siebert, Recherches sur les ateliers de bols à reliefs du Péloponnèse à l'époque hellénistique (1978) 229 ff. Taf.

90 A. Frova, JGS 13, 1971, 38 ff. Abb.; P. Donati, NumAntCl 7, 1978, 203 ff. Abb.; ders., NumAntCl 9, 1980,285 ff. Abb.; E. Roffia, Rivista di archeologica dell'antica Provincia e Diocesi di Como 163, 1981,115 ff. Taf. 1 ff.; dies., Quaderni Centro Studi Lunensi 8, 1983, 89 ff. Abb.; G. Lehrer Jacobson, JGS 34, 1992,35 ff. Abb.; M. Sternini in: The Inscribed Economy. Proceedings of a Conference, Rome 1992 (1993) 81 ff. Abb.

91 Vgl. allgemein G. Siebert, Ktema 3, 1978, 123.

92 V. Casolo, Acme 40, 1, 1987, 57 ff. Abb.; D. Kassab, Statuettes en terre cuite de Myrina. Corpus des signatures, monogrammes, lettres et signes (1988) passim; G. Nachtergael in: Tranquillitas. Mélanges en l'honneur de Tran tam Tinh (1994) 413 ff. Abb.

93 G. Schauerte, Terrakotten mütterlicher Gottheiten (1985) 56 ff.; H. Lange, KölnJb 27, 1994,142 ff. Abb.

94 L. Mercando in: EAA Suppl. 1970 (1973) 419 ff. s. v. lucerna mit zahllosen Belegen; E. Buchi, Lucerne del Museo di Aquileia I. Lucerne romane con marchio di fabbrica (1975) passim; D. M. Bailey, A Catalogue of the Lamps in the British Museum II (1980) $89 \mathrm{ff}$. 
Unterseite gewählt, da nur hier genügend Platz für die Anbringung einer Inschrift vorhanden war.

Erinnert sei abschließend daran, daß sich ein vergleichbares Phänomen, Signaturen an versteckter Stelle anzubringen, auch in der neueren Kunstgeschichte nachweisen läßt ${ }^{95}$.

Apl. Prof. Dr. Michael Donderer

Archäologisches Institut, Universität Erlangen-Nürnberg, Kochstraße 4/19, D-91054 Erlangen

Abbildungsnachweis: Abb. 1: Inst.Neg. Rom 68.3468; Abb. 2: Museumsphoto; Abb. 3. 4: Inst.Neg. Rom 76.1992; 76.1993; Abb. 5-8: Museumsphotos; Abb. 9: Forschungszentrum Griechisch-Römisches Ägypten, Universität Trier; Abb. 10-13: Museumsphotos; Abb. 14: Photo Verf.; Abb. 15: Inst.Neg. Athen 86/94; Abb. 16: nach Ancient Portraiture (1992) 227 Abb. 4; Abb. 17: Photo G. Koch; Abb. 18: Photo M. Prange; Abb. 19. 20: Photos Verf.; Abb. 21. 22: Inst.Neg. Istanbul R26401; R26398+399; Abb. 23. 24: nach AntPl VII (1967) Taf. 24 Abb. 1.

95 H. Vollmer, Die Kunst 21, 1910, 568; H. Huth, Künstler und Werkstatt der Spätgotik (1923) 66 ff. mit Belegen in Anm. 127; M. J. Liebmann in: Lucas Cranach. Künstler und Gesellschaft. Referate des Colloquiums zum 500. Geburtstag Lucas Cranach d.Ä., Wittenberg 1972 (1973) 129 f.; F. Perrot, Revue de l'Art 26, 1974, 33 ff. 